\title{
LOW MACH NUMBER LIMIT FOR THE COMPRESSIBLE MAGNETOHYDRODYNAMIC EQUATIONS IN A BOUNDED DOMAIN FOR ALL TIME*
}

\author{
CHANGSHENG DOU ${ }^{\dagger}$ AND QIANGCHANG JU๋
}

\begin{abstract}
We verify the low Mach number limit of global smooth solutions to the compressible magnetohydrodynamic equations in a bounded smooth domain in $\mathbb{R}^{2}$ with perfectly conducting boundary is verified for all time, provided that the initial data are well-prepared.
\end{abstract}

Key words. Compressible MHD equations, low Mach number limit, perfectly conducting boundary.

AMS subject classifications. 76N99, 35M33, 35Q30.

\section{Introduction}

Magnetohydrodynamics (MHD) studies the dynamics of compressible quasineutrally ionized fluids under the influence of electromagnetic fields, and has a very broad range of applications. In the present paper, we consider the flow in a perfectly conducting container which is assumed to be a bounded and connected domain $\Omega \subset \mathbb{R}^{2}$ with smooth boundary. We shall study the initial boundary value problem of the following resistive magnetohydrodynamic equations of a compressible viscous conducting fluid:

$$
\begin{aligned}
& \partial_{t} \rho+\operatorname{div}(\rho \mathbf{u})=0, \\
& \partial_{t}(\rho \mathbf{u})+\operatorname{div}(\rho \mathbf{u} \otimes \mathbf{u})+\frac{1}{\epsilon^{2}} \nabla p(\rho)=\operatorname{div}(2 \mu \mathrm{D}(\mathbf{u}))+\lambda \nabla \operatorname{div} \mathbf{u}+(\nabla \times \mathbf{H}) \times \mathbf{H}, \\
& \partial_{t} \mathbf{H}-\nabla \times(\mathbf{u} \times \mathbf{H})=-\nabla \times(\eta \nabla \times \mathbf{H}), \quad \operatorname{div} \mathbf{H}=0 .
\end{aligned}
$$

Here $\rho, \mathbf{u}=\left(u_{1}, u_{2}\right)$, and $\mathbf{H}=\left(H_{1}, H_{2}\right)$ denote the density, the velocity, and the magnetic field of the fluid, respectively, and $\mathrm{D}(\mathbf{u})=\left(\nabla \mathbf{u}+\nabla \mathbf{u}^{t}\right) / 2$. The constants $\mu$ and $\lambda$ are the shear and bulk viscosity coefficients of the fluid which satisfy $\mu>0$ and $\mu+\lambda \geq 0$; the constant $\eta>0$ is the magnetic diffusivity acting as a magnetic diffusion coefficient of the magnetic field, and $\epsilon$ is the Mach number. The pressure $P$ satisfies

$$
p(\rho)=a \rho^{\gamma}
$$

in the case of isentropic flows, where $a>0$ and $\gamma>1$ are constants.

The initial data for the system (1.1)-(1.3) are prescribed as

$$
\rho(t=0)=\rho_{0}(x), \quad \mathbf{u}(t=0)=\mathbf{u}_{0}(x), \quad \mathbf{H}(t=0)=\mathbf{H}_{0}(x) .
$$

The velocity and the magnetic field are supposed to satisfy the non-slip boundary condition and the slip boundary condition on the boundary:

$$
\mathbf{u}=0 \quad \text { on } \partial \Omega
$$

\footnotetext{
${ }^{*}$ Received: October 17, 2012; accepted (in revised form): May 20, 2013. Communicated by Zhouping Xin.

${ }^{\dagger}$ School of Statistics, Capital University of Economics and Business, Beijing 100070, P.R. China; Institute of Applied Physics and Computational Mathematics, P.O. Box 8009, Beijing 100088, P.R. China (douchangsheng@163.com).

${ }^{\ddagger}$ Institute of Applied Physics and Computational Mathematics, P.O. Box 8009, Beijing 100088, P.R. China (qiangchang_ju@yahoo.com, ju_qiangchang@iapcm.ac.cn).
} 
and

$$
\operatorname{curl} \mathbf{H}=0, \mathbf{H} \cdot \mathbf{n}=0 \text { on } \partial \Omega,
$$

where curl $\mathbf{H}=\partial_{1} H_{2}-\partial_{2} H_{1}$ and $\mathbf{n}$ is the normal vector on $\partial \Omega$. The condition (1.7) implies that the container $\Omega$ is perfectly conducting ([32]).

Recently, when the non-slip boundary condition (1.6) is replaced by the Navier slip boundary condition, Jiang and the authors proved in [9] the global existence and uniqueness of smooth solutions to the system (1.1)-(1.3), and verified the low mach number limit for all time if the initial data are well-prepared. The aim of the present paper is to extend the results in [9] to the case that the velocity is supplemented with the Dirichlet boundary condition (1.6).

The MHD equations have been studied by many applied mathematicians because of its physical importance, complexity, rich phenomena, and mathematical challenges; see, for example, $[5,10,11,16,17,26,29,36,39]$ and the references cited therein on the physical background, the well-posedness, and the vanishing viscosity limit. Recently, Jiang, Ju, Li, and Xin investigated the low Mach number limit of local smooth solutions to the full MHD equations with heat conductivity in $[21,22]$ in the whole space or a torus. The existence of global weak solutions to the MHD equations was established by [18, 37], while the low Mach number limit was studied in [19, 20]. We remark that the low Mach number limit established in [19]-[22] for the MHD equations is for the whole space or a torus, and consequently no boundary terms are involved in uniform a priori estimates. In [19], the authors also considered the limit for weak solutions in a bounded domain with some additional unusual geometry conditions.

As for the related compressible Navier-Stokes system (the system (1.1)-(1.3) with $\mathbf{H} \equiv 0$ ), we also mention that the global smooth small solutions were obtained, for example, in [34] for the non-slip boundary condition and in [40] for the Navier slip boundary condition, while the existence of global large weak solutions was established in $[13,24,25,30]$ and others. The corresponding low mach number limit was investigated extensively in $[2,3,4,6,7,8,12,15,23,27,28,31,33,35]$, and in the references cited therein.

In the following, we shall consider the flow with small density variation, i.e.,

$$
\rho=1+\epsilon \sigma
$$

Applying the usual vorticity identities together with the constraint $\operatorname{div} \mathbf{H}=0$, we can rewrite the problem (1.1)-(1.6) in the form

$$
\begin{gathered}
\partial_{t} \sigma+\operatorname{div}(\sigma \mathbf{u})+\frac{1}{\epsilon} \operatorname{div} \mathbf{u}=0, \\
\rho\left(\partial_{t} \mathbf{u}+\mathbf{u} \cdot \nabla \mathbf{u}\right)+\frac{1}{\epsilon} p^{\prime}(1+\epsilon \sigma) \nabla \sigma \\
=\mu \triangle \mathbf{u}+(\mu+\lambda) \nabla \operatorname{div} \mathbf{u}+(\mathbf{H} \cdot \nabla) \mathbf{H}-\frac{1}{2} \nabla|\mathbf{H}|^{2}, \\
\partial_{t} \mathbf{H}+(\operatorname{div} \mathbf{u}) \mathbf{H}+(\mathbf{u} \cdot \nabla) \mathbf{H}-(\mathbf{H} \cdot \nabla) \mathbf{u}=\eta \triangle \mathbf{H}, \quad \operatorname{div} \mathbf{H}=0,
\end{gathered}
$$

and the initial and boundary condition are as follows:

$$
\sigma(t=0)=\sigma_{0}(x), \quad \mathbf{u}(t=0)=\mathbf{u}_{0}(x), \quad \mathbf{H}(t=0)=\mathbf{H}_{0}(x),
$$




$$
\begin{aligned}
& \mathbf{u}=0, \quad \text { on } \partial \Omega, \\
& \operatorname{curl} \mathbf{H}=0, \mathbf{n} \cdot \mathbf{H}=0 \text { on } \partial \Omega .
\end{aligned}
$$

Thus, the main results of the present paper read as follows.

THEOREM 1.1. Let $\Omega \subset \mathbb{R}^{2}$ be a bounded domain with $C^{4}$ boundary $\partial \Omega$. There exists a positive constant $\alpha$ such that if the initial data $\sigma_{0}, \mathbf{u}_{0}$, and $\mathbf{H}_{0}$ satisfy

$$
\left\|\left(\sigma_{0}, \mathbf{u}_{0}, \mathbf{H}_{0}\right)\right\|_{\mathbf{H}^{2}}+\left\|\left(\sigma_{t}, \mathbf{u}_{t}, \mathbf{H}_{t}\right)(0)\right\|_{H^{1}} \leq \alpha
$$

with

$$
\int_{\Omega} \sigma_{0} d x=0 \text { and } 1+\epsilon \sigma_{0} \geq m \quad \text { for some constant } m>0 \text {, }
$$

and the compatibility conditions

$$
\mathbf{u}_{0}=\mathbf{H}_{0} \cdot \mathbf{n}=\operatorname{curl} \mathbf{H}_{0}=0, \text { on } \partial \Omega
$$

hold, then for any $\epsilon \in\left(0, \epsilon_{1}\right]$ where $0<\epsilon_{1}<1$ is some constant, the initial boundary value problem (1.8)-(1.13) admits a unique solution $(\sigma, \mathbf{u}, \mathbf{H})$ in $\Omega \times \overline{\mathbb{R}}^{+}$, satisfying

$$
\begin{aligned}
& \sigma \in C\left(\overline{\mathbb{R}}^{+} ; H^{2}\right), \quad(\mathbf{u}, \mathbf{H}) \in C\left(\overline{\mathbb{R}}^{+} ; H^{2}\right) \cap L^{2}\left(\overline{\mathbb{R}}^{+} ; H^{3}\right), \\
& \sigma_{t} \in C\left(\overline{\mathbb{R}}^{+} ; H^{1}\right), \quad\left(\mathbf{u}_{t}, \mathbf{H}_{t}\right) \in C\left(\overline{\mathbb{R}}^{+} ; H^{1}\right) \cap L^{2}\left(\overline{\mathbb{R}}^{+} ; H^{2}\right),
\end{aligned}
$$

where $\overline{\mathbb{R}}^{+}=[0,+\infty)$. Furthermore, it holds that

$$
\sup _{0 \leq s \leq t}\left(\|\sigma(s)\|_{H^{2}}+\|(\mathbf{u}, \mathbf{H})(s)\|_{H^{1}}+\left\|\left(\sigma_{t}, \mathbf{u}_{t}, \mathbf{H}_{t}\right)(s)\right\|_{L^{2}}\right) \leq C, \quad \forall t \in \mathbb{R}^{+}
$$

where $C$ is a positive constant independent of $\epsilon$.

THEOREM 1.2. Let the assumptions in Theorem 1.1 be satisfied, and let $(\mathbf{u}, \mathbf{H})$ be the global solution established in Theorem 1.1. Assume the initial data $\left(\mathbf{u}_{0}, \mathbf{H}_{0}\right) \rightarrow\left(\mathbf{v}_{0}, \mathbf{B}_{0}\right)$ as $\epsilon \rightarrow 0$ in $L^{2}(\Omega)$. Then $(\mathbf{u}, \mathbf{H}) \rightarrow(\mathbf{v}, \mathbf{B})$ in $C\left(\overline{\mathbb{R}}_{\mathrm{loc}}^{+} ; L^{2}(\Omega)\right)$ as $\epsilon \rightarrow 0$, and there exists a function $P(x, t)$ such that $(\mathbf{v}, \mathbf{B}, P)$ is the unique smooth solution of the following initial boundary value problem for the incompressible magnetohydrodynamic equations:

$$
\begin{aligned}
& \mathbf{v}_{t}+\mathbf{v} \cdot \nabla \mathbf{v}+\nabla P=\mu \triangle \mathbf{v}+\mathbf{B} \cdot \nabla \mathbf{B}-\frac{1}{2} \nabla|\mathbf{B}|^{2}, \quad \operatorname{div} \mathbf{v}=0, \\
& \mathbf{B}_{t}+\mathbf{v} \cdot \nabla \mathbf{B}-\mathbf{B} \cdot \nabla \mathbf{v}=\eta \triangle \mathbf{B}, \quad \operatorname{div} \mathbf{B}=0,
\end{aligned}
$$

with initial and boundary conditions

$$
\begin{aligned}
& \mathbf{v}(x, 0)=\mathbf{v}_{0}(x), \quad \mathbf{B}(x, 0)=\mathbf{B}_{0}(x), \quad x \in \Omega, \\
& \mathbf{v}=0, \quad \mathbf{B} \cdot \mathbf{n}=\operatorname{curl} \mathbf{B}=0 \quad \text { on } \partial \Omega .
\end{aligned}
$$

In the next section we shall prove theorems 1.1 and 1.2. Roughly speaking, theorems 1.1 and 1.2 are proved based on the uniform estimates of solutions in Sobolev norms which do not depend on time $t$ and the Mach number $\epsilon$. As mentioned above, compared with the Cauchy or spatially periodic problem, the presence of boundary here gives rise to some difficulties involved with controlling the boundary terms, in particular for the low Mach number limit. Moreover the techniques used in [9] for slip boundary conditions are not adequate for this case. To overcome such difficulties, the crucial step is to get the $H^{2}$-estimates of divu near the boundary, for which we shall 
adopt the local isothermal coordinates introduced in [38, 40]. This strategy has also been used in $[3,23]$ to study the low Mach limit of the compressible Navier-Stokes system with non-slip boundary condition. Compared with $[3,23]$, we need new techniques to get the estimates of magnetic field near the boundary. One key observation is that $\Delta \mathbf{H}=-\overrightarrow{\text { curl curl }} \mathbf{H}$, with $\overrightarrow{\operatorname{curl}}=\left(\partial_{2},-\partial_{1}\right)^{t}$ and $\operatorname{curl} \mathbf{u}=\partial_{1} H_{2}-\partial_{2} H_{1}$. Another is that the boundary condition (1.13) is in fact a "complementary boundary condition" in the sense of Agmon, Douglis, and Nirenberg, thus the classical theory for elliptic system is available for the magnetic field.

REMARK 1.3. When the domain $\Omega$ is three dimensional, the boundary condition (1.7) takes the form

$$
\mathbf{n} \times(\nabla \times \mathbf{H})=0 \text { on } \partial \Omega .
$$

For this case, we cannot apply directly the arguments in the present paper to get the uniform estimates of solutions, and we leave this problem for future work. On the other hand, in three dimensions when $\mathbf{H}$ satisfies the non-slip boundary condition, we can also obtain similar results as in theorems 1.1 and 1.2 by modifying the arguments in the present paper.

Before ending this section, we give the notations used throughout this paper. We use the letter $C$ (or $C_{\delta}$ ) to denote various positive constants independent of $\epsilon$ (or to emphasize the dependence on $\delta$ ). For simplicity, we denote by $H^{m}$ and $\|\cdot\|_{H^{m}}$ the standard Sobolev space $H^{m}(\Omega)$ and its norm, by $L^{p}$ and $\|\cdot\|_{L^{p}}$ the Lebesgue space $L^{p}(\Omega)$ and its norm.

\section{Proof of Theorem 1.1 and Theorem 1.2}

To prove Theorem 1.1, we first establish the local existence for the problem (1.8)(1.13) with an arbitrary but fixed $\epsilon$. Assume that the assumptions in Theorem 1.1 are satisfied. Then modifying the arguments in [38], one can show that there exists a $T^{\star}>$ 0 such that for $T \leq T^{\star}$ the problem (1.8)-(1.13) admits a unique solution satisfying

$$
\begin{aligned}
& \sigma \in C\left([0, T], H^{2}\right), \quad(\mathbf{u}, \mathbf{H}) \in C\left([0, T], H^{2}\right) \cap L^{2}\left(0, T ; H^{3}\right), \\
& \sigma_{t} \in C\left([0, T], H^{1}\right), \quad\left(\mathbf{u}_{t}, \mathbf{H}_{t}\right) \in C\left([0, T], H^{1}\right) \cap L^{2}\left(0, T ; H^{2}\right) .
\end{aligned}
$$

In the proof, it is important to note that the boundary conditions (1.13) are "complementing" boundary conditions in the sense of Agmon-Douglis-Nirenberg [1]. This fact can be verified as in [1]. Therefore the regularity theory of elliptic systems can be used in the proof. We omit the details of the proof of the local existence here.

To extend the local solution globally in time, we shall establish a differential inequality which provides us the uniform estimates of solutions for both time and the Mach number. Suppose that $(\sigma, \mathbf{u}, \mathbf{H})$ is the local solution to the initial boundary value problem $(1.8)-(1.13)$ in $\Omega \times(0, T)$, for $0<T<\infty$. Moreover, we assume that $1 / c \leq \rho=1+\epsilon \sigma \leq c$ for some constant $c>1$.

First, we obtain from the continuity equation (1.8) and the boundary condition $\mathbf{u}=0$ that

$$
\int_{\Omega} \sigma d x=\int_{\Omega} \sigma_{0} d x=0
$$

Lemma 2.1. For the solution to (1.8)-(1.13), we have

$$
\frac{1}{2} \frac{d}{d t}\left(\left\|\sqrt{p^{\prime}(1)} \sigma\right\|_{L^{2}}^{2}+\|\sqrt{\rho} \mathbf{u}\|_{L^{2}}^{2}+\|\mathbf{H}\|_{L^{2}}^{2}\right)+\gamma_{0}\left(\|\mathbf{u}\|_{H^{1}}^{2}+\|\mathbf{H}\|_{H^{1}}^{2}\right)
$$




$$
\leq C\|\mathbf{u}\|_{H^{1}}\left(\|\sigma\|_{H^{1}}^{2}+\|\mathbf{H}\|_{H^{1}}^{2}\right),
$$

where $\gamma_{0}$ and $C$ are positive constants independent of $\epsilon$.

Proof. Throughout this section we denote the inner product in $L^{2}(\Omega)$ by

$$
\langle f, g\rangle:=\int_{\Omega} f g d x .
$$

By taking $\left\langle(1.8), p^{\prime}(1) \sigma\right\rangle$, we see that

$$
\frac{1}{2} \frac{d}{d t}\left\|\sqrt{p^{\prime}(1)} \sigma\right\|_{L^{2}}^{2}-\frac{p^{\prime}(1)}{\epsilon} \int_{\Omega} \mathbf{u} \cdot \nabla \sigma d x=-p^{\prime}(1) \int_{\Omega} \sigma \operatorname{div}(\sigma \mathbf{u}) d x \leq C\|\mathbf{u}\|_{H^{1}}\|\sigma\|_{H^{1}}^{2} .
$$

Integrating by parts and using the boundary condition (1.12), one gets

$$
\left.-\int_{\Omega}(\operatorname{div}(2 \mu \mathrm{D}(\mathbf{u}))+\lambda \nabla \operatorname{div} \mathbf{u}) \cdot \mathbf{u} d x=\left.\int_{\Omega}(2 \mu \mid \mathrm{D}(\mathbf{u}))\right|^{2}+\lambda(\operatorname{div} \mathbf{u})^{2}\right) d x \geq \gamma_{0}\|\mathbf{u}\|_{H^{1}}^{2}
$$

for some constant $\gamma_{0}>0$. Thus, we take $\langle(1.9), \mathbf{u}\rangle$ to derive that

$$
\begin{aligned}
& \frac{1}{2} \frac{d}{d t}\|\sqrt{\rho} \mathbf{u}\|_{L^{2}}^{2}+\frac{p^{\prime}(1)}{\epsilon} \int_{\Omega} \mathbf{u} \cdot \nabla \sigma d x+\gamma_{0}\|\mathbf{u}\|_{H^{1}}^{2} \\
\leq & \int_{\Omega} \frac{p^{\prime}(1)-p^{\prime}(1+\epsilon \sigma)}{\epsilon} \nabla \sigma \cdot \mathbf{u} d x+\int_{\Omega}\left((\mathbf{H} \cdot \nabla) \mathbf{H}-\frac{1}{2} \nabla|\mathbf{H}|^{2}\right) \mathbf{u} d x \\
\leq & C\left(\|\mathbf{u}\|_{H^{1}}\|\sigma\|_{H^{1}}^{2}+\|\mathbf{u}\|_{H^{1}}\|\mathbf{H}\|_{H^{1}}^{2}\right) .
\end{aligned}
$$

To deal with the magnetic field equations, we denote $\overrightarrow{\operatorname{curl}}=\left(\partial_{2},-\partial_{1}\right)^{t}$. Then, the equation (1.10) can be written as

$$
\partial_{t} \mathbf{H}+(\operatorname{div} \mathbf{u}) \mathbf{H}+(\mathbf{u} \cdot \nabla) \mathbf{H}-(\mathbf{H} \cdot \nabla) \mathbf{u}=-\eta \overrightarrow{\operatorname{curl}} \operatorname{curl} \mathbf{H} .
$$

Taking $\langle(2.2), \mathbf{H}\rangle$ and using (1.13), we find that

$$
\begin{aligned}
& \frac{1}{2} \frac{d}{d t}\|\mathbf{H}\|_{L^{2}}^{2}+\eta\|\operatorname{curl} \mathbf{H}\|_{L^{2}}^{2}=\int_{\Omega}[(\operatorname{div} \mathbf{u}) \mathbf{H}+(\mathbf{u} \cdot \nabla) \mathbf{H}-(\mathbf{H} \cdot \nabla) \mathbf{u}] \mathbf{H} d x \\
\leq & C\|u\|_{H^{1}}\|\mathbf{H}\|_{H^{1}}^{2} .
\end{aligned}
$$

Putting the above estimates together and keeping in mind that

$$
\|\mathbf{F}\|_{H^{1}} \leq C\|\nabla \mathbf{F}\|_{L^{2}} \leq C\left(\|\operatorname{div} \mathbf{F}\|_{L^{2}}+\|\operatorname{curl} \mathbf{F}\|_{L^{2}}\right),
$$

for any vector $\mathbf{F} \in H^{1}(\Omega)$ with $\mathbf{F} \cdot \mathbf{n}=0$, we obtain the estimate (2.1).

The momentum equation (1.9) can be written as an inhomogeneous Stokes system with non-slip boundary condition:

$$
\left\{\begin{aligned}
& -\mu \Delta \mathbf{u}+\frac{p^{\prime}(1) \nabla \sigma}{\epsilon} \\
= & \frac{p^{\prime}(1)-p^{\prime}(1+\epsilon \sigma)}{\epsilon} \nabla \sigma+\lambda \nabla \operatorname{div} \mathbf{u}-\rho\left(\mathbf{u}_{t}+\mathbf{u} \cdot \nabla \mathbf{u}\right)+(\mathbf{H} \cdot \nabla) \mathbf{H}-\frac{1}{2} \nabla|\mathbf{H}|^{2}, \\
& \operatorname{div} \mathbf{u}=\operatorname{div} \mathbf{u} \\
\left.\mathbf{u}\right|_{\partial \Omega}=0 &
\end{aligned}\right.
$$


Thus we utilize the standard estimates ([14]) of the steady Stokes problem to obtain the following lemma.

Lemma 2.2. There exists a constant $C>0$, such that

$$
\|\mathbf{u}\|_{H^{3}}^{2}+\left\|\frac{\sigma}{\epsilon}\right\|_{H^{2}}^{2} \leq C\left(\|\sigma\|_{H^{2}}^{4}+\|\mathbf{u}\|_{H^{1}}^{2}\|\mathbf{u}\|_{H^{3}}^{2}+\left\|\nabla \mathbf{u}_{t}\right\|_{L^{2}}^{2}+\|\operatorname{div} \mathbf{u}\|_{H^{2}}^{2}+\|\mathbf{H}\|_{H^{1}}^{2}\|\mathbf{H}\|_{H^{3}}^{2}\right),
$$

and

$$
\|\mathbf{u}\|_{H^{3}}^{2}+\|\sigma\|_{H^{2}}^{2} \leq C\left(\|\sigma\|_{H^{2}}^{4}+\|\mathbf{u}\|_{H^{1}}^{2}\|\mathbf{u}\|_{H^{3}}^{2}+\left\|\nabla \mathbf{u}_{t}\right\|_{L^{2}}^{2}+\|\operatorname{div} \mathbf{u}\|_{H^{2}}^{2}+\|\mathbf{H}\|_{H^{1}}^{2}\|\mathbf{H}\|_{H^{3}}^{2}\right) .
$$

Since the boundary condition (1.13) is also a "complementary boundary condition" in the sense of Agmon, Douglis, and Nirenberg [1], the classical theory for elliptic equations yields the following lemma.

Lemma 2.3. There exists a constant $C>0$, such that

$$
\|\mathbf{H}\|_{H^{3}}^{2} \leq C\left(\left\|\mathbf{H}_{t}\right\|_{H^{1}}^{2}+\|\mathbf{u}\|_{H^{1}}^{2}\|\mathbf{H}\|_{H^{3}}^{2}+\|\mathbf{H}\|_{H^{1}}^{2}\|\mathbf{u}\|_{H^{3}}^{2}\right) .
$$

Now, we have to derive the estimates of the first order temporal and spatial derivatives of $(\sigma, \mathbf{u}, \mathbf{H})$.

Lemma 2.4. For the solution to (1.8)-(1.13), we have

$$
\begin{aligned}
& \frac{1}{2} \frac{d}{d t}\left[\mu\|\nabla \mathbf{u}\|_{L^{2}}^{2}+(\mu+\lambda)\|\operatorname{div} \mathbf{u}\|_{L^{2}}^{2}+\eta\|\operatorname{curl} \mathbf{H}\|_{L^{2}}^{2}\right] \\
& \quad+\frac{d}{d t} \int_{\Omega} \rho \mathbf{u}_{t} \mathbf{u} d x+\left\|\sqrt{p^{\prime}(1)} \sigma_{t}\right\|_{L^{2}}^{2}+\left\|\mathbf{H}_{t}\right\|_{L^{2}}^{2} \\
& \leq C\left(\left\|\mathbf{u}_{t}\right\|_{H^{1}}^{2}+\left\|\mathbf{u}_{t}\right\|_{H^{1}}\|\mathbf{u}\|_{H^{1}}^{2}+\left\|\sigma_{t}\right\|_{H^{1}}\|\sigma\|_{H^{1}}\|\mathbf{u}\|_{H^{1}}+\left\|\mathbf{H}_{t}\right\|_{H^{1}}\|\mathbf{H}\|_{H^{1}}\|\mathbf{u}\|_{H^{1}}\right)
\end{aligned}
$$

Proof. First, differentiating (1.9) with respect to $t$ and multiplying the resulting equations by $\mathbf{u}$ in $L^{2}$, integrating by parts and using the boundary condition (1.12), we deduce that

$$
\begin{aligned}
& \frac{1}{2} \frac{d}{d t}\left[\left(\mu\|\nabla \mathbf{u}\|_{L^{2}}^{2}+(\mu+\lambda)\|\operatorname{div} \mathbf{u}\|_{L^{2}}^{2}\right)\right]+\frac{d}{d t} \int_{\Omega} \rho \mathbf{u}_{t} \mathbf{u} d x+\frac{p^{\prime}(1)}{\epsilon} \int_{\Omega} \nabla \sigma_{t} \cdot \mathbf{u} d x \\
=\int_{\Omega}[ & \left.\frac{p^{\prime}(1)-p^{\prime}(1+\epsilon \sigma)}{\epsilon} \nabla \sigma\right]_{t} \cdot \mathbf{u} d x+\int_{\Omega}\left(\rho \mathbf{u}_{t}^{2}-\rho\left(\mathbf{u}_{t} \cdot \nabla \mathbf{u}+\mathbf{u} \cdot \nabla \mathbf{u}_{t}\right)\right) \cdot \mathbf{u} d x \\
& \quad+\int_{\Omega}\left(\mathbf{H} \cdot \nabla \mathbf{H}-\frac{1}{2} \nabla|\mathbf{H}|^{2}\right)_{t} \cdot \mathbf{u} d x \\
\leq & C\left(\left\|\mathbf{u}_{t}\right\|_{L^{2}}^{2}+\left\|\mathbf{u}_{t}\right\|_{H^{1}}\|\mathbf{u}\|_{H^{1}}^{2}+\left\|\sigma_{t}\right\|_{H^{1}}\|\sigma\|_{H^{1}}\|\mathbf{u}\|_{H^{1}}+\left\|\mathbf{H}_{t}\right\|_{H^{1}}\|\mathbf{H}\|_{H^{1}}\|\mathbf{u}\|_{H^{1}}\right) .
\end{aligned}
$$

We apply $\left\langle(1.8), p^{\prime}(1) \sigma_{t}\right\rangle$ and $\left\langle(2.2), \mathbf{H}_{t}\right\rangle$ to infer that

$$
\left\|\sqrt{p^{\prime}(1) \sigma_{t}}\right\|_{L^{2}}^{2}+\frac{p^{\prime}(1)}{\epsilon} \int_{\Omega} \sigma_{t} \operatorname{div} \mathbf{u} d x \leq C\left\|\sigma_{t}\right\|_{H^{1}}\|\sigma\|_{H^{1}}\|\mathbf{u}\|_{H^{1}}
$$

and

$$
\begin{aligned}
\frac{\eta}{2} \frac{d}{d t}\|\operatorname{curl} \mathbf{H}\|_{L^{2}}^{2}+\left\|\mathbf{H}_{t}\right\|_{L^{2}}^{2} & =\int_{\Omega}(\mathbf{H} \operatorname{div} \mathbf{u}+\mathbf{u} \cdot \nabla \mathbf{H}-\mathbf{H} \cdot \nabla \mathbf{u}) \cdot \mathbf{H}_{t} d x \\
& \leq C\|\mathbf{u}\|_{H^{1}}\|\mathbf{H}\|_{H^{1}}\left\|\mathbf{H}_{t}\right\|_{H^{1}}
\end{aligned}
$$


respectively. Summing up the above estimates and using the boundary condition (1.12) again, we obtain the lemma.

Lemma 2.5. For the solution to (1.8)-(1.13), we have

$$
\begin{aligned}
\frac{d}{d t}\|\nabla \sigma\|_{H^{1}}^{2} \leq & C_{\delta}\left(\|\sigma\|_{H^{2}}^{4}+\|\mathbf{u}\|_{H^{1}}^{2}\|\mathbf{u}\|_{H^{3}}^{2}+\left\|\nabla \mathbf{u}_{t}\right\|_{L^{2}}^{2}+\|\operatorname{div} \mathbf{u}\|_{H^{2}}^{2}\right. \\
& \left.\left.+\|\mathbf{H}\|_{H^{1}}^{2}\|\mathbf{H}\|_{H^{3}}^{2}\right)\right)+\delta\|\mathbf{u}\|_{H^{3}}^{2}, 0<\delta<1 .
\end{aligned}
$$

Proof. First, we take $\nabla(1.8)$ to get that

$$
\nabla \sigma_{t}+\nabla \operatorname{div}(\sigma \mathbf{u})+\frac{1}{\epsilon} \nabla \operatorname{div} \mathbf{u}=0 .
$$

We perform $\langle(2.10), \nabla \sigma\rangle$ to obtain that

$$
\begin{aligned}
& \frac{1}{2} \frac{d}{d t}\|\nabla \sigma\|_{L^{2}}^{2}=-\int_{\Omega}((\mathbf{u} \cdot \nabla) \nabla \sigma+\nabla \mathbf{u} \cdot \nabla \sigma+\nabla \sigma \operatorname{div} \mathbf{u}+\sigma \nabla \operatorname{div} \mathbf{u}) \cdot \nabla \sigma d x \\
& \quad-\frac{1}{\epsilon} \int_{\Omega} \nabla \operatorname{div} \mathbf{u} \cdot \nabla \sigma d x \\
& \leq C\left(\|\mathbf{u}\|_{H^{1}}\|\sigma\|_{H^{2}}^{2}+\|\nabla \operatorname{div} \mathbf{u}\|_{L^{2}}\|\sigma\|_{H^{2}}^{2}\right)+C_{\delta}\left\|\frac{\nabla \sigma}{\epsilon}\right\|_{L^{2}}^{2}+\delta\|\nabla \operatorname{div} \mathbf{u}\|_{L^{2}}^{2}
\end{aligned}
$$

for some $0<\delta<1$.

We differentiate (1.8) twice with respect to $x$ to have

$$
\nabla^{2} \sigma_{t}+(\mathbf{u} \cdot \nabla) \nabla^{2} \sigma+2 \nabla(\mathbf{u} \cdot \nabla) \nabla \sigma+\nabla^{2}(\mathbf{u} \cdot \nabla) \sigma+\nabla^{2}(\sigma \operatorname{div} \mathbf{u})+\frac{1}{\epsilon} \nabla^{2} \operatorname{div} \mathbf{u}=0 .
$$

Taking $\left\langle(2.12), \nabla^{2} \sigma\right\rangle$ and using Sobolev's and Young's inequalities, one obtains

$$
\frac{1}{2} \frac{d}{d t}\left\|\nabla^{2} \sigma\right\|_{L^{2}}^{2} \leq \delta\|\mathbf{u}\|_{H^{3}}^{2}+C_{\delta}\left(\|\sigma\|_{H^{2}}^{4}+\left\|\frac{\nabla^{2} \sigma}{\epsilon}\right\|_{L^{2}}^{2}\right) .
$$

for some $0<\delta<1$.

Combining (2.11) and (2.13) with (2.4), one gets the estimate (2.9).

Lemma 2.6. For the solution to (1.8)-(1.13), we have

$$
\begin{aligned}
& \frac{d}{d t}\left(\left\|\sqrt{p^{\prime}(1)} \sigma_{t}\right\|_{L^{2}}^{2}+\left\|\sqrt{\rho} \mathbf{u}_{t}\right\|_{L^{2}}^{2}+\left\|\mathbf{H}_{t}\right\|_{L^{2}}^{2}\right)+\gamma_{2}\left(\left\|\mathbf{u}_{t}\right\|_{H^{1}}^{2}+\left\|\operatorname{curl} \mathbf{H}_{t}\right\|_{L^{2}}^{2}\right) \\
& \leq C_{\delta}\left(\left\|\sigma_{t}\right\|_{H^{1}}^{2}\left(\left\|\mathbf{u}_{t}\right\|_{L^{2}}^{2}+\|\mathbf{u}\|_{H^{1}}^{2}+\|\mathbf{u}\|_{H^{1}}^{4}+\|\sigma\|_{H^{2}}^{2}+\left\|\sigma_{t}\right\|_{H^{1}}^{2}\right)\right. \\
& \quad+C_{\delta}\left(\left\|\mathbf{u}_{t}\right\|_{H^{1}}^{2}\|\mathbf{u}\|_{H^{1}}^{2}+\left\|\mathbf{H}_{t}\right\|_{H^{1}}^{2}\|\mathbf{H}\|_{H^{1}}^{2}+\left\|\mathbf{H}_{t}\right\|_{H^{1}}^{4}+C \epsilon^{2}\left\|\sigma_{t}\right\|_{L^{2}}^{2}+\delta\|\mathbf{u}\|_{H^{1}}^{2},\right.
\end{aligned}
$$

where $0<\delta<1$, and $\gamma_{2}$ is a positive constant independent of $\epsilon$.

Proof. Taking $\left\langle\partial_{t}(1.8), p^{\prime}(1) \sigma_{t}\right\rangle$, we get

$$
\begin{aligned}
& \frac{1}{2} \frac{d}{d t}\left\|\sqrt{p^{\prime}(1)} \sigma_{t}\right\|_{L^{2}}^{2}+\frac{p^{\prime}(1)}{\epsilon} \int_{\Omega} \sigma_{t} \operatorname{div} \mathbf{u}_{t} d x \\
= & -p^{\prime}(1) \int_{\Omega}\left(\mathbf{u} \cdot \nabla \sigma_{t}+\mathbf{u}_{t} \cdot \nabla \sigma+\sigma_{t} \operatorname{div} \mathbf{u}+\sigma \operatorname{div} \mathbf{u}_{t}\right) \sigma_{t} d x \\
\leq & \delta\left(\left\|\mathbf{u}_{t}\right\|_{H^{1}}^{2}+\|\mathbf{u}\|_{H^{1}}^{2}\right)+C_{\delta}\left(\|\sigma\|_{H^{1}}^{4}+\left\|\sigma_{t}\right\|_{H^{1}}^{4}\right),
\end{aligned}
$$


for some $0<\delta<1$, while taking $\left\langle(1.9)_{t}, \mathbf{u}_{t}\right\rangle$ and using the boundary conditions (1.12), we find that

$$
\begin{gathered}
\quad \frac{1}{2} \frac{d}{d t}\left\|\sqrt{\rho} \mathbf{u}_{t}\right\|_{L^{2}}^{2}+\mu\left\|\nabla \mathbf{u}_{t}\right\|_{L^{2}}^{2}+(\mu+\lambda)\left\|\operatorname{div} \mathbf{u}_{t}\right\|_{L^{2}}^{2}+\frac{p^{\prime}(1)}{\epsilon} \int_{\Omega} \nabla \sigma_{t} \cdot \mathbf{u}_{t} d x \\
=\int_{\Omega}\left[\frac{p^{\prime}(1)-p^{\prime}(1+\epsilon \sigma)}{\epsilon} \nabla \sigma\right]_{t} \cdot \mathbf{u}_{t}-\int_{\Omega}\left[\rho_{t} \mathbf{u}_{t}+\epsilon \sigma_{t} \mathbf{u} \cdot \nabla \mathbf{u}+\rho(\mathbf{u} \cdot \nabla \mathbf{u})_{t}\right. \\
\left.\quad+\frac{1}{2} \nabla\left(|\mathbf{H}|^{2}\right)_{t}-H_{t} \cdot \nabla H-H \cdot \nabla H_{t}\right] \cdot \mathbf{u}_{t} d x \\
\leq \delta\left\|\mathbf{u}_{t}\right\|_{H^{1}}^{2}+C_{\delta}\left(\left\|\sigma_{t}\right\|_{H^{1}}^{2}\left(\left\|\left(\mathbf{u}_{t}, \nabla \sigma\right)\right\|_{L^{2}}^{2}+\|\mathbf{u}\|_{H^{1}}^{4}\right)+\left\|\mathbf{u}_{t}\right\|_{H^{1}}^{2}\|\mathbf{u}\|_{H^{1}}^{2}\right. \\
\left.+\left\|\mathbf{H}_{t}\right\|_{H^{1}}^{2}\|\mathbf{H}\|_{H^{1}}^{2}\right)+C \epsilon^{2}\left\|\sigma_{t}\right\|_{L^{2}}^{2},
\end{gathered}
$$

for some $0<\delta<1$.

Differentiating (2.2) with respect to $t$, we obtain that

$$
\begin{aligned}
& \mathbf{H}_{t t}+\operatorname{div} \mathbf{u}_{t} \mathbf{H}+\operatorname{div} \mathbf{u} \mathbf{H}_{t}+\mathbf{u}_{t} \cdot \nabla \mathbf{H}+\mathbf{u} \cdot \nabla \mathbf{H}_{t}-\mathbf{H}_{t} \cdot \nabla \mathbf{u}-\mathbf{H} \cdot \nabla \mathbf{u}_{t} \\
= & -\eta \operatorname{curl} \operatorname{curl} \mathbf{H}_{t} .
\end{aligned}
$$

Taking $\left\langle(2.15), \mathbf{H}_{t}\right\rangle$ and using the boundary conditions (1.13), for some $0<\delta<1$, one has that

$$
\begin{aligned}
& \frac{1}{2} \frac{d}{d t}\left\|\mathbf{H}_{t}\right\|_{L^{2}}^{2}+\eta\left\|\operatorname{curl}_{\mathbf{H}_{t}}\right\|_{L^{2}}^{2} \\
= & -\int_{\Omega}\left(\operatorname{div} \mathbf{u}_{t} \mathbf{H}+\operatorname{div} \mathbf{u} \mathbf{H}_{t}+\mathbf{u}_{t} \cdot \nabla \mathbf{H}+\mathbf{u} \cdot \nabla \mathbf{H}_{t}-\mathbf{H}_{t} \cdot \nabla \mathbf{u}-\mathbf{H} \cdot \nabla \mathbf{u}_{t}\right) \cdot \mathbf{H}_{t} d x \\
\leq & \delta\left(\left\|\mathbf{u}_{t}\right\|_{H^{1}}^{2}+\|\nabla \mathbf{u}\|_{L^{2}}^{2}\right)+C_{\delta}\left(\left\|\mathbf{H}_{t}\right\|_{H^{1}}^{2}\|\mathbf{H}\|_{H^{1}}^{2}+\left\|\mathbf{H}_{t}\right\|_{H^{1}}^{4}\right) .
\end{aligned}
$$

Hence, by choosing $\delta$ appropriately small, we obtain the estimate (2.14).

Putting the estimates $(2.1),(2.5),(2.6),(2.7),(2.9)$, and (2.14) together in an appropriate way, we prove the following lemma.

LEMmA 2.7. There exists a constant $C>0$ such that

$$
\frac{d}{d t} \Phi_{0}(t)+\Psi_{0}(t) \leq C \Psi_{0}(t)\left(\Phi_{0}(t)+\Phi_{0}^{2}(t)\right)+\|\operatorname{div} \mathbf{u}\|_{H^{2}}^{2},
$$

where

$$
\begin{aligned}
\Phi_{0}(t)= & \|\mathbf{u}\|_{L^{2}}^{2}+\|\nabla \mathbf{u}\|_{L^{2}}^{2}+\|\operatorname{div} \mathbf{u}\|_{L^{2}}^{2}+\left\|\sqrt{\rho} \mathbf{u}_{t}\right\|_{L^{2}}^{2}+\|\sigma\|_{L^{2}}^{2}+\|\nabla \sigma\|_{H^{1}}^{2} \\
& +\left\|\sigma_{t}\right\|_{L^{2}}^{2}+\|\mathbf{H}\|_{L^{2}}^{2}+\|\nabla \mathbf{H}\|_{L^{2}}^{2}+\left\|\mathbf{H}_{t}\right\|_{L^{2}}^{2}+\int_{\Omega} \rho \mathbf{u}_{t} \cdot \mathbf{u} d x, \\
\Psi_{0}(t)= & \|\mathbf{u}\|_{H^{3}}^{2}+\left\|\mathbf{u}_{t}\right\|_{H^{1}}^{2}+\|\sigma\|_{H^{2}}^{2}+\left\|\sigma_{t}\right\|_{L^{2}}^{2}+\left\|\mathbf{H}_{t}\right\|_{H^{1}}^{2}+\|\mathbf{H}\|_{H^{3}}^{2} .
\end{aligned}
$$

It is clear that the crucial step is to estimate $\|\operatorname{div} \mathbf{u}\|_{H^{2}}^{2}$. As in $[38,3,23]$, we shall obtain the interior and boundary estimates of $\|\operatorname{div} \mathbf{u}\|_{H^{2}}^{2}$, respectively. Let us begin with the interior estimates. Let $\chi_{0} \in C_{0}^{\infty}(\Omega)$.

Lemma 2.8. For the smooth solution $(\sigma, \mathbf{u}, \mathbf{H})$ to the system (1.8)-(1.13), we have

$$
\frac{1}{2} \frac{d}{d t}\left\|\chi_{0} \sqrt{\rho} \nabla \mathbf{u}\right\|_{L^{2}}^{2}+\frac{1}{2} p^{\prime}(1) \frac{d}{d t}\left\|\chi_{0} \nabla \sigma\right\|_{L^{2}}^{2}+\mu\left\|\chi_{0} \nabla^{2} \mathbf{u}\right\|_{L^{2}}^{2}+\lambda\left\|\chi_{0} \nabla \operatorname{div} \mathbf{u}\right\|_{L^{2}}^{2}
$$




$$
\begin{gathered}
\leq \delta\left(\|\mathbf{u}\|_{H^{3}}^{2}+\|\sigma\|_{H^{2}}^{2}\right)+C_{\delta}\left(\|\sigma\|_{H^{1}}^{4}+\|\mathbf{u}\|_{H^{1}}^{2}\|\mathbf{u}\|_{H^{3}}^{2}+\|\nabla \mathbf{u}\|_{L^{2}}^{2}+\left\|\mathbf{u}_{t}\right\|_{L^{2}}^{2}\right. \\
\left.+\|\nabla \mathbf{u}\|_{L^{2}}^{2}\|\mathbf{u}\|_{L^{2}}^{2}+\|\nabla \sigma\|_{L^{2}}^{2}\left\|\mathbf{u}_{t}\right\|_{L^{2}}^{2}+\|\mathbf{u}\|_{H^{3}}^{2}\|\mathbf{u}\|_{H^{1}}^{4}+\|\mathbf{H}\|_{H^{1}}^{2}\right)
\end{gathered}
$$

for some $0<\delta<1$.

Proof. By taking $\left\langle(2.10), \chi_{0}^{2} p^{\prime}(1) \nabla \sigma\right\rangle+\left\langle(1.9),-\chi_{0}^{2} \triangle \mathbf{u}\right\rangle$, we eliminate the singular terms to obtain that

$$
\begin{gathered}
\frac{1}{2} \frac{d}{d t}\left\|\chi_{0} \sqrt{\rho} \nabla \mathbf{u}\right\|_{L^{2}}^{2}+\frac{1}{2} p^{\prime}(1) \frac{d}{d t}\left\|\chi_{0} \nabla \sigma\right\|_{L^{2}}^{2}+\mu\left\|\chi_{0} \nabla^{2} \mathbf{u}\right\|_{L^{2}}^{2}+\lambda\left\|\chi_{0} \nabla \operatorname{div} \mathbf{u}\right\|_{L^{2}}^{2} \\
\leq-\int_{\Omega}((\mathbf{u} \cdot \nabla) \nabla \sigma+\nabla \mathbf{u} \nabla \sigma+\nabla \sigma \operatorname{div} \mathbf{u}+\sigma \nabla \operatorname{div} \mathbf{u}) \chi_{0}^{2} p^{\prime}(1) \nabla \sigma d x \\
\quad+\int_{\Omega} \chi_{0} \nabla \chi_{0} \rho \mathbf{u}|\nabla \mathbf{u}|^{2}+\chi_{0} \nabla \chi_{0} \rho|\nabla \mathbf{u}|^{2}-\chi_{0}^{2} \rho \nabla \mathbf{u} \nabla \mathbf{u} \cdot \nabla \mathbf{u} \\
-2 \chi_{0} \nabla \chi_{0} \nabla \mathbf{u} \rho\left(\mathbf{u}_{t}+\mathbf{u} \cdot \nabla \mathbf{u}\right)-\epsilon \chi_{0}^{2} \nabla \mathbf{u} \nabla \sigma\left(\mathbf{u}_{t}+\mathbf{u} \cdot \nabla \mathbf{u}\right) d x \\
\quad+\int_{\Omega} 2 \chi_{0} \nabla \chi_{0} \nabla \mathbf{u}\left(\mu \Delta \mathbf{u}-\mu \nabla^{2} \mathbf{u}\right)+\left[(\mathbf{H} \cdot \nabla) \mathbf{H}-\frac{1}{2} \nabla \mathbf{H}^{2}\right] \chi_{0}^{2} \triangle \mathbf{u} d x \\
\leq \delta\left(\|\mathbf{u}\|_{H^{3}}^{2}+\|\sigma\|_{H^{2}}^{2}\right)+C_{\delta}\left(\|\sigma\|_{H^{1}}^{4}+\|\mathbf{u}\|_{H^{1}}^{2}\|\mathbf{u}\|_{H^{3}}^{2}+\|\nabla \mathbf{u}\|_{L^{2}}^{2}+\left\|\mathbf{u}_{t}\right\|_{L^{2}}^{2}\right. \\
+\|\nabla \mathbf{u}\|_{L^{2}}^{2}\|\mathbf{u}\|_{L^{2}}^{2}+\|\nabla \sigma\|_{L^{2}}^{2}\left\|\mathbf{u}_{t}\right\|_{L^{2}}^{2}+\|\mathbf{u}\|_{H^{3}}^{2}\|\mathbf{u}\|_{H^{1}}^{4}+\|\mathbf{H}\|_{H^{1}}^{2} .
\end{gathered}
$$

Lemma 2.9. For the smooth solution $(\sigma, \mathbf{u}, \mathbf{H})$ to the system (1.8)-(1.13), we have

$$
\begin{gathered}
\frac{1}{2} \frac{d}{d t}\left(\left\|\sqrt{p^{\prime}(1)} \chi_{0} \nabla^{2} \sigma\right\|_{L^{2}}^{2}+\left\|\chi_{0} \sqrt{\rho} \nabla^{2} \mathbf{u}\right\|_{L^{2}}^{2}\right)+\mu\left\|\chi_{0}\right\| \nabla^{3} \mathbf{u}\left\|_{L^{2}}^{2}+(\mu+\lambda)\right\| \chi_{0} \nabla^{2} \operatorname{div} \mathbf{u} \|_{L^{2}}^{2} \\
\leq C_{\delta}\left(\left\|\mathbf{u}_{t}\right\|_{H^{1}}^{2}\|\sigma\|_{H^{1}}^{2}+\|\mathbf{u}\|_{H^{1}}^{2}\|\mathbf{u}\|_{H^{3}}^{2}+\|\sigma\|_{H^{2}}^{2}\|\mathbf{u}\|_{H^{3}}^{2}+\|\mathbf{u}\|_{H^{1}}^{2}\|\sigma\|_{H^{2}}^{2}\right. \\
\left.+\|\sigma\|_{H^{2}}^{4}+\|\mathbf{u}\|_{H^{2}}^{2}+\|\mathbf{H}\|_{H^{2}}^{4}\right)+\delta\left(\|\mathbf{u}\|_{H^{3}}^{2}+\|\sigma\|_{H^{2}}^{2}\right),
\end{gathered}
$$

for some $0<\delta<1$.

Proof. By taking $\left\langle(2.12), \chi_{0}^{2} p^{\prime}(1) \nabla^{2} \sigma\right\rangle$, we get that

$$
\begin{aligned}
& \frac{1}{2} \frac{d}{d t} \int_{\Omega} p^{\prime}(1) \chi_{0}^{2}\left|\nabla^{2} \sigma\right|^{2} d x+\frac{1}{\epsilon} \int_{\Omega} p^{\prime}(1) \chi_{0}^{2} \nabla^{2} \operatorname{div} \mathbf{u} \nabla^{2} \sigma d x \\
= & -\int_{\Omega}\left\{p^{\prime}(1) \chi_{0}^{2} \mathbf{u} \cdot \nabla \frac{\left|\nabla^{2} \sigma\right|^{2}}{2}+2 p^{\prime}(1) \chi_{0}^{2} \nabla \mathbf{u} \cdot \nabla(\nabla \sigma) \nabla^{2} \sigma+\nabla^{2} \mathbf{u} \cdot \nabla \sigma p^{\prime}(1) \chi_{0}^{2} \nabla^{2} \sigma\right. \\
& \left.+\left(\nabla \sigma \nabla \operatorname{div} \mathbf{u}+\nabla^{2} \sigma \operatorname{div} \mathbf{u}+\sigma \nabla^{2} \operatorname{div} \mathbf{u}\right) \cdot p^{\prime}(1) \chi_{0}^{2} \nabla^{2} \nabla^{2} \sigma\right\} d x \\
\leq & \delta\|\mathbf{u}\|_{H^{3}}^{2}+C_{\delta}\|\sigma\|_{H^{2}}^{4}
\end{aligned}
$$

for some $0<\delta<1$. We differentiate (1.9) with respect to $x$ twice to have that

$$
\begin{aligned}
\rho \partial_{t} & \nabla^{2} \mathbf{u}+\rho(\mathbf{u} \cdot \nabla) \nabla^{2} \mathbf{u}+\frac{1}{\epsilon} \nabla^{2}(\nabla \sigma) \\
=-2 \epsilon & \nabla \sigma \nabla \mathbf{u}_{t}-2 \nabla(\rho \mathbf{u}) \cdot \nabla \nabla \mathbf{u}-\epsilon \nabla^{2} \sigma \mathbf{u}_{t}-\nabla^{2}(\rho \mathbf{u}) \cdot \nabla \mathbf{u} \\
& -\nabla^{2}\left(\frac{p^{\prime}(1)-p^{\prime}(1+\epsilon \mathbf{u})}{\epsilon} \nabla \sigma\right)+\mu \triangle \nabla^{2} \mathbf{u}+(\mu+\lambda) \nabla^{2} \nabla \operatorname{div} \mathbf{u} \\
& +\nabla^{2}(\mathbf{H} \cdot \nabla) \mathbf{H}+2 \nabla(\mathbf{H} \cdot \nabla) \nabla \mathbf{H}+(\mathbf{H} \cdot \nabla) \nabla^{2} \mathbf{H}-\frac{1}{2} \nabla^{3}\left(\mathbf{H}^{2}\right) .
\end{aligned}
$$


By taking $\left\langle(2.19), \chi_{0}^{2} \nabla^{2} \mathbf{u}\right\rangle$ and integrating by parts, one deduce that

$$
\begin{aligned}
& \frac{1}{2} \frac{d}{d t} \int_{\Omega} \chi_{0}^{2} \rho\left|\nabla^{2} \mathbf{u}\right|^{2} d x-\frac{1}{\epsilon} \int_{\Omega} p^{\prime}(1) \chi_{0}^{2} \nabla^{2} \operatorname{div} \mathbf{u} \nabla^{2} \sigma d x \\
& \quad+\int_{\Omega} \mu \chi_{0}^{2} \nabla^{3} \mathbf{u}+(\mu+\lambda) \chi_{0}^{2} \nabla^{2} \operatorname{div} \mathbf{u} d x \\
& \leq \delta\|\mathbf{u}\|_{H^{3}}^{2}+C_{\delta}\left(\left\|\mathbf{u}_{t}\right\|_{H^{1}}^{2}\|\sigma\|_{H^{1}}^{2}+\|\mathbf{u}\|_{H^{1}}^{2}\|\mathbf{u}\|_{H^{3}}^{2}+\|\sigma\|_{H^{2}}^{2}\|\mathbf{u}\|_{H^{3}}^{2}\right. \\
& \left.\quad+\|\mathbf{u}\|_{H^{1}}^{2}\|\sigma\|_{H^{2}}^{2}+\|\sigma\|_{H^{2}}^{4}+\|\mathbf{u}\|_{H^{2}}^{2}+\|\mathbf{H}\|_{H^{2}}^{4}\right)+\int_{\Omega} p^{\prime}(1) 2 \chi_{0} \nabla \chi_{0} \nabla^{2} \mathbf{u} \nabla^{2} \sigma d x \\
& \leq C_{\delta}\left(\left\|\mathbf{u}_{t}\right\|_{H^{1}}^{2}\|\sigma\|_{H^{1}}^{2}+\|\mathbf{u}\|_{H^{1}}^{2}\|\mathbf{u}\|_{H^{3}}^{2}+\|\sigma\|_{H^{2}}^{2}\|\mathbf{u}\|_{H^{3}}^{2}+\|\mathbf{u}\|_{H^{1}}^{2}\|\sigma\|_{H^{2}}^{2}\right. \\
& \left.\quad+\|\sigma\|_{H^{2}}^{4}+\|\mathbf{u}\|_{H^{2}}^{2}+\|\mathbf{H}\|_{H^{2}}^{4}\right)+\delta\left(\|\mathbf{u}\|_{H^{3}}^{2}+\|\sigma\|_{H^{2}}^{2}\right) .
\end{aligned}
$$

We sum up (2.18) and (2.20) and eliminate the singular terms to obtain that

$$
\begin{gathered}
\frac{1}{2} \frac{d}{d t}\left(\left\|\sqrt{p^{\prime}(1)} \chi_{0} \nabla^{2} \sigma\right\|_{L^{2}}^{2}+\left\|\chi_{0} \sqrt{\rho} \nabla^{2} \mathbf{u}\right\|_{L^{2}}^{2}\right)+\mu\left\|\chi_{0} \nabla^{3} \mathbf{u}\right\|_{L^{2}}^{2}+(\mu+\lambda)\left\|\chi_{0} \nabla^{2} \operatorname{div} \mathbf{u}\right\|_{L^{2}}^{2} \\
\leq C_{\delta}\left(\left\|\mathbf{u}_{t}\right\|_{H^{1}}^{2}\|\sigma\|_{H^{1}}^{2}+\|\mathbf{u}\|_{H^{1}}^{2}\|\mathbf{u}\|_{H^{3}}^{2}+\|\sigma\|_{H^{2}}^{2}\|\mathbf{u}\|_{H^{3}}^{2}+\|\mathbf{u}\|_{H^{1}}^{2}\|\sigma\|_{H^{2}}^{2}\right. \\
\left.+\|\sigma\|_{H^{2}}^{4}+\|\mathbf{u}\|_{H^{2}}^{2}+\|\mathbf{H}\|_{H^{2}}^{4}\right)+\delta\left(\|\mathbf{u}\|_{H^{3}}^{2}+\|\sigma\|_{H^{2}}^{2}\right)
\end{gathered}
$$

Now, we derive the boundary estimates by the method of local coordinates. We proceed essentially as in [23], but we need to deal carefully with the terms involving the magnetic field. For completeness, we elaborate the local coordinates as follows. First, one constructs the local coordinates by the isothermal coordinates $\lambda(\varphi)$ to derive an estimate near the boundary (see also [38]), where

$$
\lambda_{\varphi} \cdot \lambda_{\varphi}>0
$$

We cover the boundary $\partial \Omega$ by a finite number of bounded open sets $W^{k} \subset R^{2}, k=$ $1,2, \cdots, L$, such that for any $x \in W^{k} \cap \Omega$,

$$
x=\Lambda^{k}(\varphi, r) \equiv \lambda^{k}(\varphi)+r n\left(\lambda^{k}(\varphi)\right),
$$

where $\lambda^{k}(\varphi)$ is the isothermal coordinate and $n$ is the unit outer normal to $\partial \Omega$. Without confusion, we will omit the superscript $k$ in each $W^{k}$ for simplicity. The orthonormal system corresponding to the local coordinates can be constructed as

$$
e_{1}:=\frac{\lambda_{\varphi}}{\left|\lambda_{\varphi}\right|}, \quad e_{2}:=n(\lambda)
$$

A straightforward calculation gives

$$
J:=\operatorname{det} \frac{\partial x}{\partial(\varphi, r)}>0
$$

for sufficiently small $r$ and $J \in C^{2}$. We set the unknowns in local coordinates

$$
R(t, y):=\rho(t, \Lambda(y)), U(t, y):=\mathbf{u}(t, \Lambda(y)), V(t, y):=\mathbf{H}(t, \Lambda(y)) .
$$

Because the main difficulty of the boundary estimates lies in the dealing with singularity terms, it is enough that we only rewrite the equations (1.8), (1.9) in $[0, T] \times \tilde{\Omega}$ which include the singularity terms, where $\tilde{\Omega}:=\Lambda^{-1}(W \cap \Omega)$, as

$$
R_{t}+\frac{1}{\epsilon} a_{l j} D_{l} U^{j}=-\left(a_{l j} D_{l} R\right) U^{j}-R\left(a_{l j} D_{l} U^{j}\right),
$$




$$
\begin{aligned}
& (1+\epsilon R)\left(U_{t}^{i}+U^{j} a_{k j} D_{k} U^{i}\right)+\frac{p^{\prime}(1)}{\epsilon} a_{k i} D_{k} R \\
= & \mu a_{k j} D_{k}\left(a_{l j} D_{l} U^{i}\right)+(\mu+\lambda) a_{k i} D_{k}\left(a_{l j} D_{l} U^{j}\right) \\
& +\frac{p^{\prime}(1)-p^{\prime}(1+\epsilon R)}{\epsilon} a_{k i} D_{k} R+V^{j} a_{k j} D_{k} V^{i}-a_{i k} D_{k} V^{j} V^{j},
\end{aligned}
$$

with initial and boundary conditions

$$
\begin{aligned}
& (R, U, V)(t=0, x)=\left(R_{0}, U_{0}, V_{0}\right), \\
& U(t, x)=0, \quad \text { on } \partial \tilde{\Omega},
\end{aligned}
$$

where $a_{i j}$ is the $(i, j)$-th entry of the matrix $\operatorname{Jac}\left(\Lambda^{-1}\right)=\frac{\partial y}{\partial x}$. Clearly, $a_{i j}$ is a $C^{2}$ function, and it is easy to see that

$$
\sum_{j=1}^{2} a_{2 j} a_{2 j}=|n|^{2}=1, \quad \sum_{j=1}^{2} a_{1 j} a_{2 j}=0 .
$$

This localized system has the following properties (see also $[38,23]$ ).

Proposition 2.10. $D_{i}\left(J a_{i j}\right)=0$, for $j=1,2 ; \varsigma D_{\tau} U=0, \varsigma D_{\tau} D_{\xi} U=0$ on $\partial \tilde{\Omega}$ in the tangential directions $\tau, \xi=1$, where $\varsigma \in C_{0}^{\infty}\left(\Lambda^{-1}(W)\right)$.

Note that

$$
\left\|D_{y} U\right\|_{L^{p}(\Omega)} \leq C\left\|\nabla_{x} u\right\|_{L^{p}(\Omega)}, \quad\left\|D_{y}^{2} U\right\|_{L^{p}(\Omega)} \leq C\left\|\nabla_{x} u\right\|_{W^{1, p}(\Omega)}, 1 \leq p \leq \infty .
$$

We remark that the above inequalities apply to $R$ and $V$, too.

In view of the interpolation $\|\cdot\|_{H^{2}}^{2} \leq \delta\|\cdot\|_{H^{3}}^{2}+C_{\delta}\|\cdot\|_{H^{1}}^{2}$, the boundary estimate of $\left\|\nabla^{2} \operatorname{div} u\right\|_{L_{t}^{2}\left(L^{2}\right)}$ can be reduced to the estimate of

$$
\int_{0}^{t} \int_{\Omega} J \chi^{2}\left|D_{y}^{2}\left(a_{j i} D_{j} U^{i}\right)\right| d y d s
$$

where $\chi$ is a $C_{0}^{\infty}\left(\Lambda^{-1}(W)\right)$-function.

Lemma 2.11. $(R, U)$ satisfy the estimate

$$
\begin{aligned}
& \frac{d}{d t} \int_{\tilde{\Omega}} J \chi^{2}\left((1+\epsilon R)\left|D_{\xi \tau} U^{i}\right|^{2}+\left|D_{\xi \tau} R\right|^{2}\right) d y \\
& \quad+\mu \int_{\tilde{\Omega}} J \chi^{2} a_{k j} D_{k \xi \tau} U^{i} a_{l j} D_{l \xi \tau} U^{i} d y+(\mu+\lambda) \int_{\tilde{\Omega}} J \chi^{2} a_{k i} D_{k \xi \tau} U^{i} a_{l j} D_{l \xi \tau} U^{j} d y \\
& \leq \delta\left(\|\mathbf{u}\|_{H^{3}}^{2}+\|\sigma\|_{H^{2}}^{2}+\left\|\frac{\nabla^{2} \sigma}{\epsilon}\right\|_{H^{2}}^{2}\right)+C_{\delta}\left(\|\mathbf{u}\|_{H^{1}}^{2}+\|\sigma\|_{H^{2}}^{4}+\|\sigma\|_{H^{2}}^{2}\left\|\mathbf{u}_{t}\right\|_{H^{1}}^{2}\right. \\
& \quad+\|\sigma\|_{H^{2}}^{2}\|\mathbf{u}\|_{H^{1}}^{2}\|\mathbf{u}\|_{H^{3}}^{2}+\|\mathbf{u}\|_{H^{3}}^{2}\left(\|\sigma\|_{H^{2}}^{2}+\|\sigma\|_{H^{2}}^{4}\right) \\
& \left.\quad+\left(\|\mathbf{H}\|_{H^{1}}^{2}\|\mathbf{H}\|_{H^{2}}^{2}+\|\mathbf{H}\|_{H^{1}}^{2}\|\mathbf{H}\|_{H^{3}}^{2}\right)\right)
\end{aligned}
$$

Proof. We apply $D_{\xi \tau}$ to $(2.25)_{i}$ with $\xi, \tau$ being the tangential directions to $\partial \tilde{\Omega}$ to get that

$$
(1+\epsilon R) D_{\xi \tau} U_{t}^{i}+(1+\epsilon R) U^{j} a_{k j} D_{k \xi \tau} U^{i}+\frac{p^{\prime}(1)}{\epsilon} D_{\xi \tau}\left(a_{k i} D_{k} R\right)
$$




$$
\begin{aligned}
=\mu D_{\xi \tau} & \left(a_{k j} D_{k}\left(a_{l j} D_{l} U^{i}\right)\right)+(\mu+\lambda) D_{\xi \tau}\left(a_{k i} D_{k}\left(a_{l j} D_{l} U^{j}\right)\right) \\
& -\left[D_{\xi \tau}(1+\epsilon R) U_{t}^{i}+D_{\xi}(1+\epsilon R) D_{\tau} U_{t}^{i}+D_{\tau}(1+\epsilon R) D_{\xi} U_{t}^{i}\right. \\
& \quad+D_{\xi \tau}\left((1+\epsilon R) U^{j}\right) a_{k j} D_{k} U^{i}+D_{\xi}\left((1+\epsilon R) U^{j}\right) D_{\tau}\left(a_{k j} D_{k} U^{i}\right) \\
& \left.+D_{\tau}\left((1+\epsilon R) U^{j}\right) D_{\xi}\left(a_{k j} D_{k} U^{i}\right)\right] \\
& +D_{\xi \tau}\left(\frac{p^{\prime}(1)-p^{\prime}(1+\epsilon R)}{\epsilon} a_{k i} D_{k} R\right)+D_{\xi \tau}\left(V^{j} a_{k j} D_{k} V^{i}-a_{i k} D_{k} V^{j} V^{j}\right) .
\end{aligned}
$$

Then by multiplying the above identity by $J \chi^{2} D_{\xi \tau} U^{i}$ and integrating in $\tilde{\Omega}$, one deduces that

$$
\begin{aligned}
& \frac{1}{2} \frac{d}{d t} \int_{\tilde{\Omega}} J \chi^{2}(1+\epsilon R)\left|D_{\xi \tau} U^{i}\right|^{2} d y+\frac{p^{\prime}(1)}{\epsilon} \int_{\tilde{\Omega}} J \chi^{2} D_{\xi \tau}\left(a_{k i} D_{k} R\right) D_{\xi \tau} U^{i} d y \\
& \quad+\mu \int_{\tilde{\Omega}} J \chi^{2} a_{k j} D_{k \xi \tau} U^{i} a_{l j} D_{l \xi \tau} U^{i} d y+(\mu+\lambda) \int_{\tilde{\Omega}} J \chi^{2} a_{k i} D_{k \xi \tau} U^{i} a_{l j} D_{l \xi \tau} U^{j} d y \\
& =\frac{1}{2} \int_{\tilde{\Omega}} D_{k}\left(J \chi^{2} a_{k j}\right)(1+\epsilon R) U^{j}\left|D_{\xi \tau} U^{i}\right|^{2} d y \\
& \quad+\int_{\tilde{\Omega}} J \chi^{2} D_{\xi \tau} U^{i}\left(D_{\xi}(1+\epsilon R) D_{\tau} U_{t}^{i}+D_{\tau}(1+\epsilon R) D_{\xi} U_{t}^{i}+D_{\xi \tau}(1+\epsilon R) U_{t}^{i}\right) d y \\
& \quad+\int_{\tilde{\Omega}} J \chi^{2} D_{\xi \tau} U^{i}\left[D_{\xi}\left((1+\epsilon R) U^{j} a_{k j}\right) D_{k \tau} U^{i}+D_{\tau}\left((1+\epsilon R) U^{j} a_{k j}\right) D_{k \xi} U^{i}\right. \\
& \left.\quad+D_{\xi \tau}\left((1+\epsilon R) U^{j} a_{k j}\right) D_{k} U^{i}\right] d y \\
& \quad-\int_{\tilde{\Omega}} a_{l j} D_{\xi \tau} U^{i}\left[\mu D_{k}\left(J \chi^{2} a_{k j}\right) D_{l \xi \tau} U^{i}+\nu D_{k}\left(J \chi^{2} a_{k i}\right) D_{l \xi \tau} U^{j}\right] \\
& \quad+D_{\xi \tau} a_{l j}\left[\mu D_{l} U^{i} D_{k}\left(J \chi^{2} a_{k j} D_{\xi \tau} U^{i}\right)+(\mu+\lambda) D_{l} U^{j} D_{k}\left(J \chi^{2} a_{k i} D_{\xi \tau} U^{i}\right)\right] d y \\
& \quad+\int_{\tilde{\Omega}} J \chi^{2} D_{\xi \tau} U^{i}\left\{\mu \left[a_{k j} D_{k}\left(D_{\xi} a_{l j} D_{l \tau} U^{i}+D_{\tau} a_{l j} D_{l \xi} U^{i}\right)\right.\right. \\
& \left.\quad+D_{\xi} a_{k j} D_{k \tau}\left(a_{l j} D_{l} U^{i}\right)+D_{\tau} a_{k j} D_{k \xi}\left(a_{l j} D_{l} U^{i}\right)+D_{\xi \tau} a_{k j} D_{k}\left(a_{l j} D_{l} U^{i}\right)\right] \\
& \quad+(\mu+\lambda)\left[a_{k i} D_{k}\left(D_{\xi} a_{l j} D_{l \tau} U^{j}+D_{\tau} a_{l j} D_{l \xi} U^{j}\right)\right. \\
& \left.\left.\quad+D_{\xi} a_{k i} D_{k \tau}\left(a_{l j} D_{l} U^{j}\right)+D_{\tau} a_{k i} D_{k \xi}\left(a_{l j} D_{l} U^{j}\right)+D_{\xi \tau} a_{k i} D_{k}\left(a_{l j} D_{l} U^{j}\right)\right]\right\} d y \\
& \quad-\int_{\tilde{\Omega}} J \chi^{2} D_{\xi \tau} U^{i} D_{\xi \tau}\left(\frac{p^{\prime}(1)-p^{\prime}(1+\epsilon R)}{\epsilon} a_{k i} D_{k} R\right) d y \\
& +\int_{\tilde{\Omega}} J \chi^{2} D_{\xi \tau} U^{i} D_{\xi \tau}\left(V^{j} a_{k j} D_{k} V^{i}-a_{i k} D_{k} V^{j} V^{j}\right) d y=\sum_{i=1}^{7} E_{i},
\end{aligned}
$$

where each term on the right-hand side can be estimated by Gagliardo-Nirenberg's and Young's inequalities as follows:

$$
\begin{aligned}
& \left|E_{1}\right| \leq C\|\mathbf{u}\|_{H^{2}}^{2} \leq \delta\|\mathbf{u}\|_{H^{3}}^{2}+C_{\delta}\|\mathbf{u}\|_{H^{1}}^{2}, \\
& \left|E_{2}\right| \leq C\left\|\nabla^{2} \mathbf{u}\right\|_{L^{6}}\|\sigma\|_{L^{3}}\left\|\mathbf{u}_{t}\right\|_{H^{1}} \leq C\left\|\nabla^{2} \mathbf{u}\right\|_{H^{1}}\|\nabla \sigma\|_{L^{2}}^{\frac{1}{2}}\|\nabla \sigma\|_{L^{6}}^{\frac{1}{2}}\left\|\mathbf{u}_{t}\right\|_{H^{1}} \\
& \quad \leq \delta\|\mathbf{u}\|_{H^{3}}^{2}+C_{\delta}\|\sigma\|_{H^{2}}^{2}\left\|\mathbf{u}_{t}\right\|_{H^{1}}^{2} \\
& \quad+E_{3} \mid \leq C\|\nabla \sigma\|_{L^{3}}\|\mathbf{u}\|_{L^{\infty}}\left\|\nabla^{2} \mathbf{u}\right\|_{L^{2}}\left\|\nabla^{2} \mathbf{u}\right\|_{L^{6}}+C\|\nabla \mathbf{u}\|_{L^{2}}\left\|\nabla^{2} \mathbf{u}\right\|_{L^{3}}\left\|\nabla^{2} \mathbf{u}\right\|_{L^{6}}, \\
& \quad+C\left\|\nabla^{2} \sigma\right\|_{L^{2}}\|\mathbf{u}\|_{L^{3}}\|\nabla \mathbf{u}\|_{L^{\infty}}\left\|\nabla^{2} \mathbf{u}\right\|_{L^{6}}+C\|\nabla \sigma\|_{L^{3}}\|\nabla \mathbf{u}\|_{L^{\infty}}\|\nabla \mathbf{u}\|_{L^{2}}\left\|\nabla^{2} \mathbf{u}\right\|_{L^{6}}, \\
& \quad \leq \delta\|\mathbf{u}\|_{H^{3}}^{2}+C_{\delta}\|\sigma\|_{H^{2}}^{2}\|\mathbf{u}\|_{H^{1}}^{2}\|\mathbf{u}\|_{H^{3}}^{2},
\end{aligned}
$$


$\left|E_{4}\right|+\left|E_{5}\right| \leq C\|\mathbf{u}\|_{H^{2}}\|\mathbf{u}\|_{H^{3}} \leq \delta\|\mathbf{u}\|_{H^{3}}^{2}+C_{\delta}\|\mathbf{u}\|_{H^{1}}^{2}$,

and

$$
\begin{aligned}
\left|E_{6}\right| \leq C\left\|\nabla^{2} \mathbf{u}\right\|_{L^{6}}\|\nabla \sigma\|_{L^{6}}^{2}\|\nabla \sigma\|_{L^{2}}+C\left\|\nabla^{3} \mathbf{u}\right\|_{L^{2}}\|\sigma\|_{L^{\infty}}\left\|\nabla^{2} \sigma\right\|_{L^{2}} \\
\quad+C\left\|\nabla^{2} \mathbf{u}\right\|_{L^{6}}\|\nabla \sigma\|_{L^{3}}\|\sigma\|_{L^{\infty}}\left\|\nabla^{2} \sigma\right\|_{L^{2}}+C\left\|\nabla^{2} \mathbf{u}\right\|_{L^{3}}\|\nabla \sigma\|_{L^{6}}\left\|\nabla^{2} \sigma\right\|_{L^{2}}, \\
\quad \leq \delta\|\sigma\|_{H^{2}}^{2}+C_{\delta}\|\mathbf{u}\|_{H^{3}}^{2}\left(\|\sigma\|_{H^{2}}^{2}+\|\sigma\|_{H^{2}}^{4}\right) \\
\left|E_{7}\right| \leq C\left\|\nabla^{2} \mathbf{u}\right\|_{L^{6}}\left(\|\nabla \mathbf{H}\|_{L^{2}}\|\nabla \mathbf{H}\|_{L^{3}}+\|\mathbf{H}\|_{L^{3}}\left\|\nabla^{3} \mathbf{H}\right\|_{L^{2}}\right) . \\
\leq \delta\|\mathbf{u}\|_{H^{3}}^{2}+C_{\delta}\left(\|\mathbf{H}\|_{H^{1}}^{2}\|\mathbf{H}\|_{H^{2}}^{2}+\|\mathbf{H}\|_{H^{1}}^{2}\|\mathbf{H}\|_{H^{3}}^{2}\right) .
\end{aligned}
$$

We remark here that in the above estimate of $E_{6}$, we have used integration by parts in order to deal with the presence of third-order derivatives of $R$. Therefore, we conclude that

$$
\begin{aligned}
& \frac{1}{2} \frac{d}{d t} \int_{\tilde{\Omega}} J \chi^{2}(1+\epsilon R)\left|D_{\xi \tau} U^{i}\right|^{2} d y+\frac{p^{\prime}(1)}{\epsilon} \int_{\tilde{\Omega}} J \chi^{2} D_{\xi \tau}\left(a_{k i} D_{k} R\right) D_{\xi \tau} U^{i} d y \\
& \quad+\mu \int_{\tilde{\Omega}} J \chi^{2} a_{k j} D_{k \xi \tau} U^{i} a_{l j} D_{l \xi \tau} U^{i} d y+(\mu+\lambda) \int_{\tilde{\Omega}} J \chi^{2} a_{k i} D_{k \xi \tau} U^{i} a_{l j} D_{l \xi \tau} U^{j} d y \\
& \leq \delta\left(\|\mathbf{u}\|_{H^{3}}^{2}+\|\sigma\|_{H^{2}}^{2}\right)+C_{\delta}\left(\|\mathbf{u}\|_{H^{1}}^{2}+\|\sigma\|_{H^{2}}^{2}\left\|\mathbf{u}_{t}\right\|_{H^{1}}^{2}+\|\sigma\|_{H^{2}}^{2}\|\mathbf{u}\|_{H^{1}}^{2}\|\mathbf{u}\|_{H^{3}}^{2}\right. \\
& \left.\quad+\|\mathbf{u}\|_{H^{3}}^{2}\left(\|\sigma\|_{H^{2}}^{2}+\|\sigma\|_{H^{2}}^{4}\right)+\left(\|\mathbf{H}\|_{H^{1}}^{2}\|\mathbf{H}\|_{H^{2}}^{2}+\|\mathbf{H}\|_{H^{1}}^{2}\|\mathbf{H}\|_{H^{3}}^{2}\right)\right) .
\end{aligned}
$$

In order to eliminate the singular term on the left-hand side of (2.31), we argue, similarly to the above procedure, to find that

$$
\begin{aligned}
& \frac{1}{2} \frac{d}{d t} \int_{\tilde{\Omega}} p^{\prime}(1) J \chi^{2}\left|D_{\xi \tau} R\right|^{2} d y-\frac{p^{\prime}(1)}{\epsilon} \int_{\tilde{\Omega}} J \chi^{2} D_{\xi \tau}\left(a_{k i} D_{k} R\right) D_{\xi \tau} U^{i} d y \\
=\int_{\tilde{\Omega}} & \frac{p^{\prime}(1)}{\epsilon}\left\{D_{l}\left(J \chi^{2} a_{l j}\right) D_{\xi \tau} U^{j} D_{\xi \tau} R-\left[D_{\xi}\left(a_{l j}\right) D_{l \tau} U^{j}+D_{\tau}\left(a_{l j}\right) D_{l \xi} U^{j}\right.\right. \\
& \left.+D_{\xi \tau}\left(a_{l j}\right) D_{l} U^{j}\right] J \chi^{2} D_{\xi \tau} R-J \chi^{2} D_{\xi \tau} U^{j}\left[D_{\xi}\left(a_{l j}\right) D_{l \tau} R\right. \\
& \left.\left.+D_{\tau}\left(a_{l j}\right) D_{l \xi} R+D_{\xi \tau}\left(a_{l j}\right) D_{l} R\right]\right\} d y-\int_{\tilde{\Omega}} p^{\prime}(1) J \chi^{2} D_{\xi \tau}\left(a_{l j} D_{l} R U^{j}\right) D_{\xi \tau} R d y \\
& -\int_{\tilde{\Omega}} p^{\prime}(1) J \chi^{2} D_{\xi \tau}\left(R a_{l j} D_{l} U^{j}\right) D_{\xi \tau} R d y:=\sum_{i=1}^{3} I_{i},
\end{aligned}
$$

where the terms $I_{i}$ can be bounded as follows, using Sobolev's inequality and Lemma 2.2:

$$
\begin{aligned}
I_{1} & \leq C\left\|\nabla^{2} \mathbf{u}\right\|_{L^{2}}\left(\left\|\frac{\nabla^{2} \sigma}{\epsilon}\right\|_{L^{2}}+\left\|\frac{\nabla \sigma}{\epsilon}\right\|_{L^{2}}\right) \leq \delta\left(\left\|\frac{\nabla^{2} \sigma}{\epsilon}\right\|_{H^{2}}^{2}+\|\mathbf{u}\|_{H^{3}}^{2}\right)+C_{\delta}\|\mathbf{u}\|_{H^{1}}^{2}, \\
I_{2} & \leq C\left(\|\nabla \mathbf{u}\|_{L^{\infty}}\left\|\nabla^{2} \sigma\right\|_{L^{2}}^{2}+\|\nabla \sigma\|_{L^{3}}\left\|\nabla^{2} \mathbf{u}\right\|_{L^{6}}\left\|\nabla^{2} \sigma\right\|_{L^{2}} \leq \delta\|\mathbf{u}\|_{H^{3}}^{2}+C_{\delta}\|\sigma\|_{H^{2}}^{4},\right. \\
I_{3} & \leq C\left(\|\nabla \mathbf{u}\|_{L^{\infty}}\left\|\nabla^{2} \sigma\right\|_{L^{2}}^{2}+\|\nabla \sigma\|_{L^{3}}\left\|\nabla^{2} \mathbf{u}\right\|_{L^{6}}\left\|\nabla^{2} \sigma\right\|_{L^{2}}+\|\sigma\|_{L^{\infty}}\left\|\nabla^{3} \mathbf{u}\right\|_{L^{2}}\left\|\nabla^{2} \sigma\right\|_{L^{2}}\right) \\
& \leq \delta\|\mathbf{u}\|_{H^{3}}^{2}+C_{\delta}\|\sigma\|_{H^{2}}^{4} .
\end{aligned}
$$

Hence, we have

$$
\frac{1}{2} \frac{d}{d t} \int_{\tilde{\Omega}} p^{\prime}(1) J \chi^{2}\left|D_{\xi \tau} R\right|^{2} d y-\frac{p^{\prime}(1)}{\epsilon} \int_{\tilde{\Omega}} J \chi^{2} D_{\xi \tau}\left(a_{k i} D_{k} R\right) D_{\xi \tau} U^{i} d y
$$




$$
\leq \delta\left(\left\|\frac{\nabla^{2} \sigma}{\epsilon}\right\|_{H^{2}}^{2}+\|\mathbf{u}\|_{H^{3}}^{2}\right)+C_{\delta}\left(\|\mathbf{u}\|_{H^{1}}^{2}+\|\sigma\|_{H^{2}}^{4}\right) .
$$

Combining (2.31) with (2.32), one deduces the estimate (2.30).

Next we turn to the estimate of the derivatives in the normal direction. According to the idea in Valli's paper [38], we will deal with the components of higher-order derivatives in the normal direction to $\partial \tilde{\Omega}$. Taking $a_{2 i}$ as in (2.25), we get that

$$
\begin{aligned}
& (2 \mu+\lambda) D_{2}\left(a_{l j} D_{l} U^{j}\right)-\frac{p^{\prime}(1)}{\epsilon} D_{2} R \\
=(1+\epsilon & R)\left(U_{t}^{i}+U^{j} a_{k j} D_{k} U^{i}\right) a_{2 i} \\
& \quad-\frac{p^{\prime}(1)-p^{\prime}(1+\epsilon R)}{\epsilon} D_{2} R+\left(a_{i k} D_{k} V^{j} V^{j}-V^{j} a_{k j} D_{k} V^{i}\right) a_{2 i} \\
& +\mu\left(D_{2}\left(a_{l j} D_{l} U^{j}\right)-a_{k j} a_{2 i} D_{k}\left(a_{l j} D_{l} U^{i}\right)\right) .
\end{aligned}
$$

After a straightforward calculation, we see that

$$
\begin{aligned}
& \mu\left(D_{2}\left(a_{l j} D_{l} U^{j}\right)-a_{k j} a_{2 i} D_{k}\left(a_{l j} D_{l} U^{i}\right)\right) \\
= & \mu\left(D_{2} a_{2 j} D_{2} U^{j}+D_{2} a_{\tau j} D_{\tau} U^{j}+a_{\tau j} D_{2 \tau} U^{j}-a_{2 j} D_{2} a_{2 j} a_{2 i} D_{2} U^{i}\right. \\
& \left.\quad-a_{\tau j} a_{2 i} D_{\tau} a_{l j} D_{l} U^{i}-a_{\tau j} a_{\xi j} a_{2 i} D_{\xi \tau} U^{i}-a_{2 j} a_{2 i} D_{2} a_{\tau j} D_{\tau} U^{i}\right)
\end{aligned}
$$

for $\tau, \xi=1$, which does not include the second-order normal derivative $D_{22} U$.

First, we take the first-order derivative of $(2.33)$ with respect to $y_{\tau}(\tau=1)$, then multiply by $J \chi^{2} D_{\tau 2}\left(a_{l j} D_{l} U^{j}\right)$ in $L^{2}(\tilde{\Omega})$ to get that

$$
\begin{aligned}
& \frac{2 \mu+\lambda}{2} \int_{\tilde{\Omega}} J \chi^{2}\left|D_{\tau 2}\left(a_{l j} D_{l} U^{j}\right)\right|^{2} d y-\frac{p^{\prime}(1)}{\epsilon} \int_{\tilde{\Omega}} D_{\tau 2} R D_{\tau 2}\left(a_{l j} D_{l} U^{j}\right) d y \\
& \leq C\left(\|\nabla \sigma\|_{L^{3}}^{2}\left\|\mathbf{u}_{t}\right\|_{L^{6}}^{2}+\|\nabla \sigma\|_{L^{6}}^{2}\|\mathbf{u}\|_{L^{6}}^{2}\|\nabla \mathbf{u}\|_{L^{6}}^{2}+\left\|u_{t}\right\|_{H^{1}}^{2}+\|\nabla \mathbf{u}\|_{L^{6}}^{2}\|\nabla \mathbf{u}\|_{L^{3}}^{2}\right. \\
& \quad+\|\mathbf{u}\|_{L^{\infty}}^{2}\left\|\nabla^{2} \mathbf{u}\right\|_{L^{2}}^{2}+\|\nabla \sigma\|_{L^{6}}^{2}\|\nabla \sigma\|_{L^{3}}^{2}+\|\sigma\|_{L^{\infty}}^{2}\left\|\nabla^{2} \sigma\right\|_{L^{2}}^{2}+\|\nabla \mathbf{H}\|_{L^{6}}^{2}\|\nabla \mathbf{H}\|_{L^{3}}^{2} \\
& \left.\quad+\|\mathbf{H}\|_{L^{\infty}}^{2}\left\|\nabla^{2} \mathbf{H}\right\|_{L^{2}}^{2}+\|\mathbf{u}\|_{H^{1}}^{2}+\int_{\tilde{\Omega}} J \chi^{2}\left|D_{\tau \xi y} U\right|^{2} d y\right) \\
& \leq C\left(\|\sigma\|_{H^{2}}^{2}\left\|\nabla \mathbf{u}_{t}\right\|_{L^{2}}^{2}+\|\sigma\|_{H^{2}}^{2}\|\mathbf{u}\|_{H^{1}}^{2}\|\mathbf{u}\|_{H^{2}}^{2}+\left\|u_{t}\right\|_{H^{1}}^{2}+\|\mathbf{u}\|_{H^{1}}^{2}\right. \\
& \left.\quad+\|\mathbf{u}\|_{H^{1}}^{2}\|\mathbf{u}\|_{H^{3}}^{2}+\|\sigma\|_{H^{2}}^{4}+\|\mathbf{H}\|_{H^{1}}^{2}\|\mathbf{H}\|_{H^{3}}^{2}\right)+C \int_{\tilde{\Omega}} J \chi^{2}\left|D_{\tau \xi y} U\right|^{2} d y
\end{aligned}
$$

Correspondingly, we apply $D_{\tau 2}$ to $(2.24)$ and multiply the resulting identity by $p^{\prime}(1) J \chi^{2} D_{\tau 2} R$ in $L^{2}(\tilde{\Omega})$ to obtain that

$$
\begin{aligned}
& \frac{p^{\prime}(1)}{2} \frac{d}{d t} \int_{\tilde{\Omega}} J \chi^{2}\left|D_{\tau 2} R\right|^{2} d y+\frac{p^{\prime}(1)}{\epsilon} \int_{\tilde{\Omega}} J \chi^{2} D_{\tau 2} R D_{\tau 2}\left(a_{k j} D_{k} U^{j}\right) d y \\
= & -\int_{\tilde{\Omega}} p^{\prime}(1) J \chi^{2} D_{\tau 2}\left[\left(a_{l j} D_{l} R\right) U^{j}+R\left(a_{l j} D_{l} U^{j}\right)\right] D_{\tau 2} R d y \\
\leq & \delta\|\mathbf{u}\|_{H^{3}}^{2}+C_{\delta}\left(\|\sigma\|_{H^{2}}^{4}+\|\sigma\|_{H^{1}}^{2}\|\sigma\|_{H^{2}}^{2}\right) .
\end{aligned}
$$

Combing (2.34) with (2.35), we obtain the following lemma.

Lemma 2.12. There exists a small $\delta>0$ such that $R$ and $U$ satisfy

$$
\frac{d}{d t} \int_{\tilde{\Omega}} J \chi^{2}\left|D_{\tau 2} R\right|^{2} d y+\int_{\tilde{\Omega}} J \chi^{2}\left|D_{\tau 2}\left(a_{l j} D_{l} U^{j}\right)\right|^{2} d y
$$




$$
\begin{gathered}
\leq C\left(\|\sigma\|_{H^{2}}^{2}\left\|\nabla \mathbf{u}_{t}\right\|_{L^{2}}^{2}+\|\sigma\|_{H^{2}}^{2}\|\mathbf{u}\|_{H^{1}}^{2}\|\mathbf{u}\|_{H^{2}}^{2}+\left\|u_{t}\right\|_{H^{1}}^{2}+\|\mathbf{u}\|_{H^{1}}^{2}+\|\mathbf{u}\|_{H^{1}}^{2}\|\mathbf{u}\|_{H^{3}}^{2}\right. \\
\left.+\|\sigma\|_{H^{2}}^{4}+\|\mathbf{H}\|_{H^{1}}^{2}\|\mathbf{H}\|_{H^{3}}^{2}\right)+C \int_{\tilde{\Omega}} J \chi^{2}\left|D_{\tau \xi y} U\right|^{2} d y \\
+\delta\|\mathbf{u}\|_{H^{3}}^{2}+C_{\delta}\left(\|\sigma\|_{H^{2}}^{4}+\|\sigma\|_{H^{1}}^{2}\|\sigma\|_{H^{2}}^{2}\right) .
\end{gathered}
$$

Second, we need to estimate $\left\|D_{22}\left(a_{l j} D_{i} U^{j}\right)\right\|_{L^{2}(\tilde{\Omega})}$ to close the estimate for divu. We apply $D_{2}$ to $(2.33)$ to get

$$
\begin{aligned}
(2 \mu+ & \lambda) D_{22}\left(a_{l j} D_{l} U^{j}\right)-\frac{p^{\prime}(1)}{\epsilon} D_{22} R \\
=D_{3}( & \left.(1+\epsilon R) a_{2 i}\right)\left(U_{t}^{i}+U^{j} a_{k j} D_{k} U^{i}\right) \\
& +(1+\epsilon R) a_{2 i}\left(D_{2} U_{t}^{i}+D_{2}\left(U^{j} a_{k j} D_{k} U^{i}\right)\right)-D_{2}\left(\frac{p^{\prime}(1)-p^{\prime}(1+\epsilon R)}{\epsilon} D_{2} R\right) \\
& +D_{2}\left[\left(a_{i k} D_{k} V^{j} V^{j}-V^{j} a_{k j} D_{k} V^{i}\right) a_{2 i}\right]+O(1)\left(D_{22 \tau} U^{j}+D_{2 l} U^{j}+D_{l} U^{j}\right) .
\end{aligned}
$$

Multiplying the above identity by $J \chi^{2} D_{22}\left(a_{l j} D_{l} U^{j}\right)$ in $L^{2}(\tilde{\Omega})$, we see that

$$
\begin{aligned}
& \quad \frac{2 \mu+\lambda}{2} \int_{\tilde{\Omega}} J \chi^{2}\left|D_{22}\left(a_{l j} D_{l} U^{j}\right)\right|^{2} d y-\frac{p^{\prime}(1)}{\epsilon} \int_{\tilde{\Omega}} J \chi^{2} D_{22} R D_{22}\left(a_{l j} D_{l} U^{j}\right) d y \\
& \leq C\left(\|\sigma\|_{H^{2}}^{2}\left\|\nabla \mathbf{u}_{t}\right\|_{L^{2}}^{2}+\|\sigma\|_{H^{2}}^{2}\|\mathbf{u}\|_{H^{1}}^{2}\|\mathbf{u}\|_{H^{2}}^{2}+\left\|u_{t}\right\|_{H^{1}}^{2}+\|\mathbf{u}\|_{H^{1}}^{2}\right. \\
& \left.\quad+\|\mathbf{u}\|_{H^{1}}^{2}\|\mathbf{u}\|_{H^{3}}^{2}+\|\sigma\|_{H^{2}}^{4}+\|\mathbf{H}\|_{H^{1}}^{2}\|\mathbf{H}\|_{H^{3}}^{2}\right)+C \int_{\tilde{\Omega}} J \chi^{2}\left|D_{33 \tau} U\right|^{2} d y .
\end{aligned}
$$

Meanwhile, we apply $D_{22}$ to (2.24) and multiply the resulting identity by $p^{\prime}(1) J \chi^{2} D_{22} R$ in $L^{2}(\tilde{\Omega})$ to obtain that

$$
\begin{aligned}
& \frac{p^{\prime}(1)}{2} \frac{d}{d t} \int_{\tilde{\Omega}} J \chi^{2}\left|D_{22} R\right|^{2} d y+\frac{p^{\prime}(1)}{\epsilon} \int_{\tilde{\Omega}} J \chi^{2} D_{22} R D_{22}\left(a_{k j} D_{k} U^{j}\right) d y \\
= & -\int_{\tilde{\Omega}} p^{\prime}(1) J \chi^{2} D_{22}\left[\left(a_{l j} D_{l} R\right) U^{j}+R\left(a_{l j} D_{l} U^{j}\right)\right] D_{22} R d y \\
\leq & \delta\|\mathbf{u}\|_{H^{3}}^{2}+C_{\delta}\left(\|\sigma\|_{H^{2}}^{4}+\|\sigma\|_{H^{1}}^{2}\|\sigma\|_{H^{2}}^{2}\right) .
\end{aligned}
$$

Then, we add (2.36) to (2.37) to obtain the following.

Lemma 2.13. There exists a small constant $\delta>0$ such that $R$, and $U$ satisfy

$$
\begin{aligned}
& \quad \frac{d}{d t} \int_{\tilde{\Omega}} J \chi^{2}\left|D_{22} R\right|^{2} d y+\int_{\tilde{\Omega}} J \chi^{2}\left|D_{22}\left(a_{l j} D_{l} U^{j}\right)\right|^{2} d y \\
& \leq C\left(\|\sigma\|_{H^{2}}^{2}\left\|\nabla \mathbf{u}_{t}\right\|_{L^{2}}^{2}+\|\sigma\|_{H^{2}}^{2}\|\mathbf{u}\|_{H^{1}}^{2}\|\mathbf{u}\|_{H^{2}}^{2}+\left\|u_{t}\right\|_{H^{1}}^{2}+\|\mathbf{u}\|_{H^{1}}^{2}\right. \\
& \left.\quad+\|\mathbf{u}\|_{H^{1}}^{2}\|\mathbf{u}\|_{H^{3}}^{2}+\|\sigma\|_{H^{2}}^{4}+\|\mathbf{H}\|_{H^{1}}^{2}\|\mathbf{H}\|_{H^{3}}^{2}\right)+C \int_{\tilde{\Omega}} J \chi^{2}\left|D_{22 \tau} U\right|^{2} d y \\
& \quad+\delta\|\mathbf{u}\|_{H^{3}}^{2}+C_{\delta}\left(\|\sigma\|_{H^{2}}^{4}+\|\sigma\|_{H^{1}}^{2}\|\sigma\|_{H^{2}}^{2}\right) .
\end{aligned}
$$

Third, we have to estimate the rest of the terms in the third-order normal derivatives. As in [38], we introduce the following Stokes problem in the original coordinates in the region $W \cap \Omega$ :

$$
\left\{\begin{array}{l}
-\mu \triangle_{x}\left[\left(\chi D_{\tau} U\right) \circ \Lambda^{-1}\right]+\frac{p^{\prime}(1)}{\epsilon} \nabla_{x}\left[\left(\chi D_{\tau} R\right) \circ \Lambda^{-1}\right]=F_{1}, \\
\operatorname{div}_{x}\left[\left(\chi D_{\tau} U\right) \circ \Lambda^{-1}\right]=F_{2} \\
\left(\chi D_{\tau} U\right) \circ \Lambda^{-1}=0, \quad \text { on } \partial(W \cap \Omega),
\end{array}\right.
$$


where

$$
\begin{aligned}
F_{1}^{i}=\chi D_{\tau} & \left((1+\epsilon R) U_{t}^{i}+U^{j} a_{k j} D_{k} U^{i}-(\mu+\lambda) a_{k i} D_{k}\left(a_{l j} D_{l} U^{j}\right)\right) \\
& +\chi D_{\tau}\left(\frac{p^{\prime}(1)-p^{\prime}(1+\epsilon R)}{\epsilon} a_{k i} D_{k} R+V^{j} a_{k j} D_{k} V^{i}-a_{i k} D_{k} V^{j} V^{j}\right) \\
& +O(1)\left(D_{l} U^{i}+D_{k l} U^{i}+\frac{1}{\epsilon} D_{k} R\right),
\end{aligned}
$$

Due to the regularity theory for the Stokes system (in [14]), one deduces

$$
\int_{W \cap \Omega}\left|\triangle_{x}\left(\chi D_{\tau} U\right) \circ \Lambda^{-1}\right|^{2} d x \leq C\left(\left\|F_{1}\right\|_{L^{2}(W \cap \Omega)}^{2}+\left\|F_{2}\right\|_{H^{1}(W \cap \Omega)}^{2}\right) .
$$

By virtue of the above inequality, and the fact that $\int_{W \cap \Omega}\left|\triangle_{x}\left(\chi D_{\tau} U\right) \circ \Lambda^{-1}\right|^{2} d x$ is equivalent to

$$
\begin{aligned}
& \int_{\tilde{\Omega}} J\left|\sum_{j=1}^{2} \sum_{k=1}^{2} a_{k j} D_{k}\left(\sum_{l=1}^{2} a_{l j} D_{l}\left(\chi D_{\tau} U\right)\right)\right|^{2} d y \\
= & \int_{\tilde{\Omega}} J \chi^{2}\left|\sum_{j, k, l=1}^{2} a_{k j} a_{l j} D_{k l \tau} U\right|^{2} d y+O(1) \int_{\tilde{\Omega}}\left(\left|D_{\tau} U\right|^{2}+\left|D_{y \tau} U\right|^{2}\right) d y,
\end{aligned}
$$

and

$$
D_{22 \tau} U=\sum_{k, l=1}^{2}\left(\sum_{j=1}^{2} a_{k j} a_{l j}\right) D_{k l \tau} U-\sum_{1 \leq k, l \leq 1} \sum_{j=1}^{2} a_{k j} a_{l j} D_{k l \tau} U
$$

which follows from (2.28), we infer that

$$
\begin{aligned}
\int_{\tilde{\Omega}} J \chi^{2}\left|D_{22 \tau} U\right|^{2} d y \leq C(\| & \left.F_{1}\left\|_{L^{2}(W \cap \Omega)}^{2}+\right\| F_{2} \|_{H^{1}(W \cap \Omega)}^{2}\right) \\
& +C \int_{\tilde{\Omega}} J \chi^{2}\left|D_{\xi \tau \zeta}\right|^{2} d y+C_{\delta}\|\nabla \mathbf{u}\|_{L^{2}}^{2}+\delta\|\mathbf{u}\|_{H^{3}}^{2} .
\end{aligned}
$$

By Hölder's inequality and the interpolation inequality, we get that

$$
\begin{gathered}
\left\|F_{1}\right\|_{L^{2}}^{2} \leq C\left(\|\mathbf{u}\|_{H^{1}}^{2}+\|\mathbf{u}\|_{H^{2}}^{2}+\left\|\frac{\nabla \sigma}{\epsilon}\right\|_{L^{2}}^{2}\right) \\
+C\left(\|\sigma\|_{H^{2}}^{2}\left\|\nabla \mathbf{u}_{t}\right\|_{L^{2}}^{2}+\|\sigma\|_{H^{2}}^{2}\|\mathbf{u}\|_{H^{1}}^{2}\|\mathbf{u}\|_{H^{2}}^{2}+\left\|u_{t}\right\|_{H^{1}}^{2}+\|\mathbf{u}\|_{H^{1}}^{2}\right. \\
\left.+\|\mathbf{u}\|_{H^{1}}^{2}\|\mathbf{u}\|_{H^{3}}^{2}+\|\sigma\|_{H^{2}}^{4}+\|\mathbf{H}\|_{H^{1}}^{2}\|\mathbf{H}\|_{H^{3}}^{2}\right) \\
\left.\quad+\|\mathbf{u}\|_{H^{1}}^{2}\|\mathbf{u}\|_{H^{3}}^{2}+\|\sigma\|_{H^{2}}^{4}+\|\mathbf{H}\|_{H^{1}}^{2}\|\mathbf{H}\|_{H^{3}}^{2}\right)
\end{gathered}
$$

where we have used the $H^{2}$-estimate of the Stokes system, i.e.,

$$
\|\mathbf{u}\|_{H^{2}}^{2}+\left\|\frac{\nabla \sigma}{\epsilon}\right\|_{L^{2}}^{2} \leq C\left(\|\sigma\|_{H^{2}}^{4}+\|\mathbf{u}\|_{H^{1}}^{2}\|\mathbf{u}\|_{H^{3}}^{2}+\left\|\nabla \mathbf{u}_{t}\right\|_{L^{2}}^{2}+\|\operatorname{div} \mathbf{u}\|_{H^{1}}^{2}\right) .
$$

As for the estimate of $G_{2}$, it is easy to see that

$$
\left\|G_{2}\right\|_{H^{1}}^{2} \leq \delta\|\mathbf{u}\|_{H^{3}}^{2}+C_{\delta}\|\nabla \mathbf{u}\|_{L^{2}}^{2}+C \int_{\tilde{\Omega}} J \chi^{2}\left|D_{k \tau}\left(a_{l j} D_{l} U^{j}\right)\right|^{2} d y
$$


Substituting (2.40) and (2.41) into (2.39), we obtain

$$
\begin{array}{r}
\int_{\tilde{\Omega}} J \chi^{2}\left|D_{22 \tau} U\right|^{2} d y \leq C_{\delta}\left(\|\mathbf{u}\|_{H^{1}}^{2}+\|\sigma\|_{H^{2}}^{2}\left\|\nabla \mathbf{u}_{t}\right\|_{L^{2}}^{2}+\|\sigma\|_{H^{2}}^{2}\|\mathbf{u}\|_{H^{1}}^{2}\|\mathbf{u}\|_{H^{2}}^{2}+\left\|u_{t}\right\|_{H^{1}}^{2}\right. \\
\left.+\|\mathbf{u}\|_{H^{1}}^{2}\|\mathbf{u}\|_{H^{3}}^{2}+\|\sigma\|_{H^{2}}^{4}+\|\mathbf{H}\|_{H^{1}}^{2}\|\mathbf{H}\|_{H^{3}}^{2}\right) \\
+C \int_{\tilde{\Omega}} J \chi^{2}\left(\left|D_{\xi \tau y}\right|^{2}+\left|D_{2 \tau}\left(a_{l j} D_{l} U^{j}\right)\right|^{2}\right) d y+\delta\|\mathbf{u}\|_{H^{3}}^{2} .
\end{array}
$$

In view of lemmas 2.11-2.13 and the estimate (2.42), we have thus shown the following estimate in the normal direction.

Lemma 2.14. Denote

$$
\begin{aligned}
& \Psi_{\chi}(t):=\int_{\tilde{\Omega}} J \chi^{2}\left((1+\epsilon R)\left|D_{\xi \tau} U^{i}\right|^{2}+\left|D_{\xi \tau} R\right|^{2}+\left|D_{\tau 2} R\right|^{2}+\left|D_{22} R\right|^{2}\right)(t) d y, \\
& \Phi_{\chi}(t):=\int_{\tilde{\Omega}} J \chi^{2}\left(\left|D_{y \xi \tau} U\right|^{2}+\left|D_{\tau 2}\left(a_{l j} D_{l} U^{j}\right)\right|^{2}+\left|D_{22}\left(a_{l j} D_{l} U^{j}\right)\right|^{2}+\left|D_{22 \tau} U\right|^{2}\right)(t) d y .
\end{aligned}
$$

Then we have

$$
\begin{aligned}
\frac{d}{d t} \Psi_{\chi}(t)+\Phi_{\chi}(t) \leq \delta\left(\|\mathbf{u}\|_{H^{3}}^{2}+\|\sigma\|_{H^{2}}^{2}+\left\|\frac{\nabla^{2} \sigma}{\epsilon}\right\|_{H^{2}}^{2}\right)+C_{\delta}\left(\|\mathbf{u}\|_{H^{1}}^{2}+\left\|\mathbf{u}_{t}\right\|_{H^{1}}^{2}+\|\sigma\|_{H^{2}}^{4}\right. \\
+\|\sigma\|_{H^{2}}^{2}\left\|\mathbf{u}_{t}\right\|_{H^{1}}^{2}+\|\sigma\|_{H^{2}}^{2}\|\mathbf{u}\|_{H^{1}}^{2}\|\mathbf{u}\|_{H^{3}}^{2}+\|\mathbf{u}\|_{H^{1}}^{2}\|\mathbf{u}\|_{H^{3}}^{2} \\
\left.+\|\mathbf{u}\|_{H^{3}}^{2}\left(\|\sigma\|_{H^{2}}^{2}+\|\sigma\|_{H^{2}}^{4}\right)+\left(\|\mathbf{H}\|_{H^{1}}^{2}\|\mathbf{H}\|_{H^{2}}^{2}+\|\mathbf{H}\|_{H^{1}}^{2}\|\mathbf{H}\|_{H^{3}}^{2}\right)\right)
\end{aligned}
$$

for some $0<\delta<1$.

DeFinition 2.15 .

$$
\begin{gathered}
\Psi(t):=l\|\mathbf{u}\|_{H^{1}}^{2}+\left\|\sqrt{\rho} \mathbf{u}_{t}\right\|_{L^{2}}^{2}+\|\sigma\|_{H^{2}}^{2}+\left\|\sigma_{t}\right\|_{L^{2}}^{2} \\
+\|\mathbf{H}\|_{H^{1}}^{2}+\left\|\mathbf{H}_{t}\right\|_{L^{2}}^{2}+\int_{\Omega} \rho \mathbf{u}_{t} \cdot \mathbf{u} d x+\|\left.\left[\nabla^{2} \mathbf{u}\right]\right|_{t a n}, \\
\Phi(t):=\|\mathbf{u}\|_{H^{3}}^{2}+\left\|\mathbf{u}_{t}\right\|_{H^{1}}^{2}+\|\sigma\|_{H^{2}}^{2}+\left\|\sigma_{t}\right\|_{L^{2}}^{2}+\left\|\mathbf{H}_{t}\right\|_{H^{1}}^{2}+\|\mathbf{H}\|_{H^{3}}^{2},
\end{gathered}
$$

where $l$ is a large enough constant and $\left|\left[\nabla^{2} \mathbf{u}\right]\right|_{\text {tan }}$ is the $L^{2}$-norm of the second-order derivatives of $\mathbf{u}$ except the normal components to $\partial \Omega$.

Combining lemmas 2.1, 2.7-2.9 with Lemma 2.14, choosing $\epsilon$ and $\delta$ small enough, and transforming the local coordinates into the usual ones, we finally conclude that

$$
\frac{d}{d t} \Psi(t)+\Phi(t) \leq c_{0} \Phi(t)\left(\Psi(t)+\Psi^{2}(t)\right),
$$

where $c_{0} \geq 1$ is a constant independent of $\epsilon$.

Now, employing (2.43), and following the analysis in [38], we obtain the following uniform estimate.

Lemma 2.16. Suppose $\Psi(0) \leq \beta /\left(2 c_{0}\right)$ for some $\beta \in(0,1 / 2]$, where $c_{0}$ is the same as in (2.43). Then there is an $\epsilon_{1}>0$ such that for any $\epsilon \in\left(0, \epsilon_{1}\right]$, we have $c^{-1} \leq 1+\epsilon \sigma \leq c$ for some $c>1$, and $\Psi(t) \leq \beta /\left(2 c_{0}\right)$ for all $t \in[0, T]$.

Now, recalling the definition $(2.15)$ of $\Psi(t)$, we can use the uniform a priori estimate established in Lemma 2.16 to continue the local solution $(\sigma, \mathbf{u}, \mathbf{H})$ globally in time by applying the standard extension techniques (see, for example, [40]), and obtain 
therefore a global solution. Furthermore, we can employ the uniform estimate given in Lemma 2.16 and Arzelà-Ascoli's theorem to easily show the strong convergence of $(\sigma, \mathbf{u}, \mathbf{H})$ to the solution of the corresponding incompressible magnetohydrodynamic equations as $\epsilon \rightarrow 0$. This completes the proof of Theorem 1.1 and Theorem 1.2.

Acknowledgment. The authors would like to thank Professor Song Jiang for helpful suggestions. Ju was supported by the NSFC (Grant No. 40890154 and 11171035) and Dou by the China Postdoctoral Science Foundation (No. 2012M520205).

\section{REFERENCES}

[1] S. Agmon, A. Douglis, and L. Nirenberg, Estimates near the boundary for solutions of elliptic partial differential equations satisfying general boundary conditions, II, Commun. Pure Appl. Math., 17, 35-92, 1964.

[2] T. Alazard, Low Mach number limit of the full Navier-Stokes equations, Arch. Ration. Mech. Anal., 180, 1-73, 2006.

[3] H. Bessaih, Limite de modeles de fluides compressibles, Portugaliae Math., 52, 441-463, 1995.

[4] D. Bresch, B. Desjardins, E. Grenier, and C.K. Lin, Low Mach number limit of viscous polytropic flows: Formal asymptotics in the periodic case, Stud. Appl. Math., 109, 125-149, 2002.

[5] G.Q. Chen and D.H. Wang, Global solutions of nonlinear magnetohydrodynamics with large initial data, J. Diff. Equ., 182, 344-376, 2002.

[6] R. Danchin, Low Mach number limit for viscous compressible flows, Math. Model. Numer. Anal., 39, 459-475, 2005.

[7] B. Desjardins and E. Grenier, Low Mach number limit of viscous compressible flows in the whole space, R. Soc. Lond. Proc. Ser. A Math. Phys. Eng. Sci., 455, 2271-2279, 1999.

[8] B. Desjardins, E. Grenier, P.L. Lions, and N. Masmoudi, Incompressible limit for solutions of the isentropic Navier-Stokes equations with Dirichlet boundary conditions, J. Math. Pures Appl., 78, 461-471, 1999.

[9] C. Dou, S. Jiang, and Q. Ju, Global existence and the low Mach number limit for the compressible magnetohydrodynamic equations in a bounded domain with perfectly conducting boundary, Z. Angew. Math. Phys., DOI 10.1007/s00033-013-0311-7, 2013.

[10] B. Ducomet and E. Feireisl, The equations of magnetohydrodynamics: On the interaction between matter and radiation in the evolution of gaseous stars, Commun. Math. Phys., 266, 595-629, 2006.

[11] J. Fan, S. Jiang, and G. Nakamura, Vanishing shear viscosity limit in the magnetohydrodynamic equations, Commun. Math. Phys., 270, 691-708, 2007.

[12] E. Feireisl and A. Novotný, Singular Limits in Thermodynamics of Viscous Fluids, Birkhauser, Basel, 2009.

[13] E. Feireisl, A. Novotný, and H. Petzeltová, On the existence of globally defined weak solutions to the Navier-Stokes equations, J. Math. Fluid Mech., 3, 358-392, 2001.

[14] G.P. Galdi, An Introduction to the Mathematical Theory of the Navier-Stokes Equations. Vol. I. Linearized Steady Problems, Springer-Verlag, New York, 1994.

[15] T. Hagstrom and J. Lorenz, On the stability of approximate solutions of hyperbolic-parabolic systems and the all-time existence of smooth, slightly compressible flows, Indiana Univ. Math. J., 51, 1339-1387, 2002.

[16] D. Hoff and E. Tsyganov, Uniqueness and continuous dependence of weak solutions in compressible magnetohydrodynamics, Z. Angew. Math. Phys., 56, 791-804, 2005.

[17] W.R. Hu, Cosmic Magnetohydrodynamics, Science Press, Beijing, 1987 (in Chinese).

[18] X.P. Hu and D.H. Wang, Global existence and large-time behavior of solutions to the threedimensional equations of compressible magnetohydrodynamic flows, Arch. Ration. Mech. Anal., 197, 203-238, 2010.

[19] X.P. Hu and D.H. Wang, Low Mach number limit of viscous compressible magnetohydrodynamic flows, SIAM J. Math. Anal., 41, 1272-1294, 2009.

[20] S. Jiang, Q.C. Ju, and F.C. Li, Incompressible limit of the compressible Magnetohydrodynamic equations with periodic boundary conditions, Commun. Math. Phys., 297, 371-400, 2010.

[21] S. Jiang, Q.C. Ju, and F.C. Li, Low Mach number limit for the multi-dimensional full magnetohydrodynamic equations, Nonlin., 25, 1351-1365, 2012. 
[22] S. Jiang, Q.C. Ju, F.C. Li, and Z. P. Xin, Low Mach number limit for the multi-dimensional full mag- netohydrodynamic equations with general initial data, available at arXiv: $1111.2925 \mathrm{v} 1$.

[23] S. Jiang and Y.B. Ou, Incompressible limit of the non-isentropic Navier-Stokes equations with well-prepared initial data in three-dimensional bounded domains, J. Math. Pures Appl., 96, $1-28,2011$.

[24] S. Jiang and P. Zhang, Global spherically symmetry solutions of the compressible isentropic Navier-Stokes equations, Commun. Math. Phys., 215, 559-581, 2001.

[25] S. Jiang and P. Zhang, Axisymmetric solutions of the 3-D Navier-Stokes equations for compressible isentropic fluids, J. Math. Pures Appl., 82, 949-973, 2003.

[26] S. Kawashima, Systems of a Hyperbolic-Parabolic Composite Type, with Applications to the Equations of Magnetohydrodynamics, Ph.D Thesis, Kyota University, 1983.

[27] H. Kim and J. Lee, The incompressible limits of viscous polytropic fluids with zero thermal conductivity coeffient, Commun. PDE, 30, 1169-1189, 2005.

[28] S. Klainerman and A. Majda, Singular perturbations of quasilinear hyperbolic systems with large parameters and the incompressible limit of compressible fluids, Commun. Pure Appl. Math., 34, 481-524, 1981.

[29] L.D. Laudau and E.M. Lifshitz, Electrodynamics of Continuous Media, Second Edition, Pergamon, New York, 1984.

[30] P.L. Lions, Mathematical Topics in Fluid Mechanics, Vol. 1: Incompressible Models, Oxford Lecture Series in Mathematics and its Applications 3, The Clarendon Press and Oxford University Press, New York, 1996.

[31] P.L. Lions and N. Masmoudi, Incompressible limit for a viscous compressible fluid, J. Math. Pures Appl., 77, 585-627, 1998.

[32] J.G. Liu and R. Pego, Stable discretization of magnetohydrodynamics in bounded domains, Commun. Math. Sci., 8, 234-251, 2010.

[33] N. Masmoudi, Examples of singular limits in hydrodynamics, in: Handbook of Differential Equations: Evolutionary Equations, Vol. III, Elsevier/North-Holland, Amsterdam, 195-275, 2007.

[34] A. Matsumura and T. Nishida, Initial boundary value problems for the equations of motion of compressible viscous and heat-conductive fluids, Commun. Math. Phys., 89, 445-464, 1983.

[35] Y. Ou, Incompressible limits of the Navier-Stokes equations for all time, J. Diff. Equ., 247, 3295-3314, 2009.

[36] R.V. Polovin and V.P. Demutskii, Fundamentals of Magnetohydrodynamics, Consultants, Bureau, New York, 1990.

[37] A. Suen and D. Hoff, Global low-energy weak solutions of the equations of three-dimensional compressible magnetohyfrodynamics, Arch. Ration. Mech. Anal., 205, 27-58, 2012.

[38] A. Valli, Periodic and stationary solutions for compressible Navier-Stokes equations via a stability method, Ann. Scuola Norm. Sup. Pisa Cl. Sci., 10(4), 607-647, 1983.

[39] J.W. Zhang, S. Jiang, and F. Xie, Global weak solutions of an initial boundary value problem for screw pinches in plasma physics, Math. Mode. Meth. Appl. Sci., 19, 833-875, 2009.

[40] W.M. Zajaczkowski, On nonstationary motion of a compressible baratropic viscous fluids with boundary slip condition, J. Appl. Anal., 4, 167-204, 1998. 